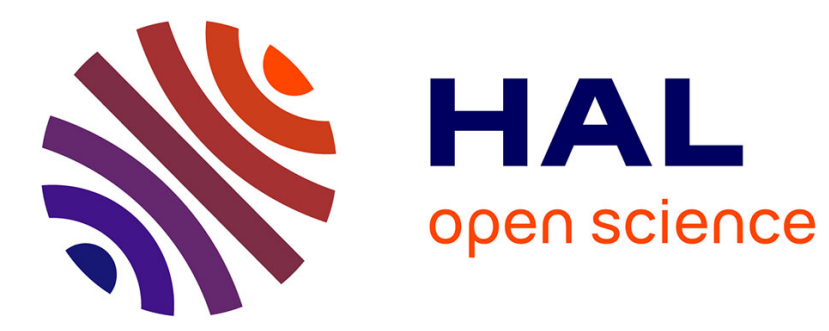

\title{
Functionalization of 2D materials by intercalation
}

Lakshya Daukiya, Maya Narayanan Nair, Marion Cranney, Francois Vonau, Samar Hajjar-Garreau, Dominique Aubel, Laurent Simon

\section{To cite this version:}

Lakshya Daukiya, Maya Narayanan Nair, Marion Cranney, Francois Vonau, Samar Hajjar-Garreau, et al.. Functionalization of 2D materials by intercalation. Progress in Surface Science, 2018, 94 (1), pp.1-20. 10.1016/j.progsurf.2018.07.001 . hal-02375289

\section{HAL Id: hal-02375289 \\ https://hal.science/hal-02375289}

Submitted on 9 Dec 2020

HAL is a multi-disciplinary open access archive for the deposit and dissemination of scientific research documents, whether they are published or not. The documents may come from teaching and research institutions in France or abroad, or from public or private research centers.
L'archive ouverte pluridisciplinaire HAL, est destinée au dépôt et à la diffusion de documents scientifiques de niveau recherche, publiés ou non, émanant des établissements d'enseignement et de recherche français ou étrangers, des laboratoires publics ou privés. 


\title{
Functionalization of 2D materials by intercalation
}

\author{
L. Daukiya, M. N. Nair, M. Cranney, F. Vonau, S. Hajjar-Garreau, D. \\ Aubel and L. Simon ${ }^{1}$ \\ Université de Haute-Alsace, CNRS, IS2M UMR 7361, 3Bis rue Alfred Werner, 68100 \\ Mulhouse, France Université de Strasbourg, France
}

\begin{abstract}
Since the discovery of graphene many studies focused on its functionalization by different methods. These strategies aim to find new pathways to overcome the main drawback of graphene, a missing band-gap, which strongly reduces its potential applications, particularly in the domain of nanoelectronics, despite its huge and unequaled charge carrier mobility. The necessity to contact this material with a metal has motivated a lot of studies of metal/graphene interactions and has led to the discovery of the intercalation process very early in the history of graphene. Intercalation, where the deposited atoms do not stay at the graphene surface but intercalate between the top layer and the substrate, may happen at room temperature or be induced by annealing, depending of the chemical nature of the metal. This kind of mechanism was already well-known in the earlier Graphite Intercalation Compounds (GICs), particularly famous for one current application, the Lithium-ion Battery, which is simply an application based on the intercalation of Lithium atoms between two sheets of graphene in a graphite anode. Among numerous discoveries the GICs community also found a way to obtain graphite with superconducting properties by using intercalated alkali metals. Graphene is now a playground to "revisit" and understand all these mechanisms and to discover possible new properties of graphene induced by intercalation. For example, the intercalation process may be used to decouple the graphene layer from its substrate, to change its doping level or even, in a more general way, to modify its electronic band structure and the nature of its Dirac fermions. In this paper we will focus on the functionalization of graphene by using inter-
\end{abstract}

1. corresponding author

E-mail address : Laurent.simon@uha.fr

Preprint submitted to Progress in surface science

3 juillet 2018

(C) 2018 published by Elsevier. This manuscript is made available under the Elsevier user license https://www.elsevier.com/open-access/userlicense/1.0/ 
calation of metal atoms but also of molecules. We will give an overview of the induced modifications of the electronic band structure possibly leading to spin-orbit coupling, superconductivity, ... We will see how this concept of functionalization is also now used in the framework of other $2 \mathrm{D}$ materials beyond graphene and of van der Waals heterostructures based on these materials.

Keywords:

\section{Nomenclature}

TMDs Transition Metal Dichalcogenides

ARPES Angle Resolved Photoelectron Spectroscopy

QPs Quasiparticles

STM Scanning Tunneling Microscopy

STS Scanning Tunneling Spectroscopy

FTSTS Fourier Transform Scanning Tunneling Spectroscopy

SiC Silicon Carbide

ML Mono layer

GICs Graphite Intercalation Compounds

DFT Density Functional Theory

$\mathrm{AuF} \mathrm{Au}$ films

IC Intercalated clusters

HOPG Highly Oriented Pyrolytic Graphite

CEC Constant Energy Contour

VHs Van Hove Singularities

$E_{f}$ Fermi level

$E_{D}$ Energy of Dirac point

SO Spin-orbit

QSHE Quantum Spin Hall Effect

UHV Ultra High Vacuum

BCS Bardeen, L. Cooper and J. Schieffer

EPC Electron Phonon Coupling

D Density of electrons

SQUID Superconducting QUantum Interference Device

XPS X-ray Photoelectron Spectroscopy

UPS UV Photoelectron Spectroscopy

LEED Low-Energy Electron Diffraction 
LEEM Low-Energy electron microscopy

MOCVD MetalOrganic Chemical Vapor Deposition

\section{Introduction}

"Simple systems are already so complicated, why take an interest in complicated systems?". We would certainly use this relevant citation for the graphene system. Indeed this 2D layer of carbon atoms arranged in a honeycomb lattice, put in light by A.K. Geim and K.S. Novoselov [1], is certainly the most "a priori" simple system that solid-state physicists started to study. The only complexity of graphene arises from its non-Bravais lattice leading to a Dirac-like linear dispersion and this permits to explore and evidence a rich unexpected physics with a lot of fascinating properties. Among numerous potential applications we will focus here on nanoelectronics and particularly recall that graphene has the highest electron mobility ever observed (up to $10^{5} \mathrm{~cm}^{2} / \mathrm{V} . \mathrm{s}$ ) [2], even at room temperature. Moreover, it has a spin relaxation length between 10 to $100 \mu \mathrm{m}$ [3] and Quantum Hall Effect was observed for the first time at room temperature on this system [4]. Despite these record-breaking performances, graphene still does not supplant silicon [5] and does not experience the expected breakthrough in the field of micro/nanoelectronics because of its lack of energy band gap. To overcome this major obstacle, several methods of functionalization have been studied, but this remains a challenge, as graphene is highly non-reactive. Deposition of species like metallic atoms, molecules, the intercalation of these elements, the patterning of graphene with templates, the cutting of graphene nanoribbon are tested as possible methods of functionalization. Furthermore, the goal is to perturb in a controlled manner the graphene lattice using a long-range perfect organization with a sub-2-3 nm periodicity [6]. All these difficulties may explain why a large part of the scientific community is turning towards other 2D materials such as Transition Metal Dichalcogenides (TMDs) [7], or is exploring the Group IV of the Periodic table with the discoveries of silicene [8, 9], germanene [10], and more recently stanene [11] which are the analogs of graphene, based on Silicon, Germanium and Tin atoms respectively. In the same way the 2D black Phosphorene [12] and even Borophene [13] were also discovered. For these materials a Dirac-like cone dispersion with a gap opening is expected but was not clearly demonstrated. Indeed, the band structure is always polluted by the band structure of the substrate as an iso- 
lated 2D layer was never really obtained [14]. In the case of the growth of Silicen on Au(111), using ARPES measurements, Oughaddou et al[15] recently reported linear Dirac cone with an opening of gap $>0.5 \mathrm{eV}$ clearly distinguished from gold sp bands. The measured Fermi velocity of $10^{6} \mathrm{~m} . \mathrm{s}^{-1}$ strongly supports the presence of Dirac fermions. However another weak point of these new 2D materials is their high reactivity and their consecutive instability even in UHV.

Because of its simple structure, graphene has become a playground to understand the Fermiology of Dirac quasiparticles (QP). Playing with Dirac fermions means to modify locally, if possible periodically, the hopping potential of the electrons between neighboring atoms in the lattice graphene. For example, theory predicts possible arising and merging of new Dirac points in the band structure [16], the opening of a gap using defects with specific symmetry perturbing the QP [6] or a strong modification of their Fermi velocity. By patterning graphene with the formation of a lattice of holes or the formation of ribbons of graphene, it is theoretically possible to induce a high anisotropy of electron mobility $[17,18]$. It is also possible to confine electrons by the modification of the coupling between two graphene layers using rotationally stacked commensurate bilayers graphene [19]. Several experimental attempts have been made to induce a periodical perturbation on graphene by patterning using self-assembled templates. The superimposing of a periodicity on Bloch waves induces a folding of the first Brillouin zone leading to an opening of a gap at the boundary of the mini-Brillouin zone, an approach based on the well-known method used in semiconductor physics $[20,21]$. With a periodicity from $100 \mathrm{~nm}$ to micrometer scale, a high conductivity and an on/off ratio up to 10 were obtained but with a gap of $50 \mathrm{meV}$ only [22]. This shows that up to now, this method is not very efficient on graphene.

Several methods of functionalization using molecules were explored. When the molecules are in van der Waals interactions with graphene, the effect on the band structure is mainly a transfer of charge with a shift of the Dirac point relative to the Fermi level [23]. This was done either by evaporation of molecules in UHV or by deposition of molecules in solvent using ex-situ methods such as drop casting or spin coating [24, 25]. There are possibly more efficient methods of molecular functionalization, which should really modify the graphene band structure. For example, covalent grafting of reactive molecule [26], fluorination using chemical method [27] or a site-selective fluorination by a local radicalization of graphene using focused ion beam 
(FIB) irradiation [28], which is a softer method compatible with electronic lithography process. Unfortunately, graphene became either completely insulating or without sizable modifications of its band structure.

Among all the functionalization methods, the intercalation of species like metallic atoms, molecules or gas, is to date the most efficient method to significantly modify the band structure of graphene, not only the Dirac point position but also the shape of the Dirac cones. It permits for example to decouple the graphene layer from its substrate, using $\mathrm{Ge}, \mathrm{Au}$ or $\mathrm{H}$ in the case of epitaxial graphene/ $\mathrm{SiC}(0001)[29,30,31]$ but also using Au intercalation for graphene $/ \mathrm{Ni}(111)[32,33]$ or upon oxygen intercalation for graphene/ $\mathrm{Ru}(0001)$ [34].

Most of these studies were performed on epitaxial graphene/ $\mathrm{SiC}(0001)$ as it is a reference system to understand the effects of functionalization, particularly by intercalation, by following the modifications of the band structure. Indeed, as the graphene layer is well oriented, the electronic band structure can be easily measured using angle resolved photoemission spectroscopy (ARPES). Moreover, as $\mathrm{SiC}$ is a large band gap semi-conductor, the conductance of the functionalized graphene is easily measured by putting contact on the graphene surface. All modifications of this epitaxial graphene can be imaged by Scanning Tunneling Microscopy (STM). Finally it is possible to control the number of graphene layers simply by changing the temperature and annealing time of the $\mathrm{SiC}$ substrate, growing homogenous samples with only the buffer layer or with 1 to 3 graphene layers on top [35, 36]. As schematized figure 1, this epitaxial graphene consists in a monolayer on top of a buffer layer (in red on the figure), also called "zerolayer graphene", which is a graphene layer partially covalently bound to the outermost silicon atoms of the substrate leading to a $(6 \sqrt{3} \times 6 \sqrt{3})$ R30 reconstruction if we refer to the G-1 $\times 1$ graphene lattice and a $6 \times 6$ reconstruction if we refer to the initial $\mathrm{SiC}-1 \times 1 \mathrm{SiC}$ lattice $[37,38,35,39]$. Only the monolayer graphene (in green in Fig. 1), which is in van der Waals interaction with the buffer layer below, has the well-known band dispersion with Dirac cones without energy band gap. A gap of $0.5 \mathrm{eV}$ has been observed after the partial decoupling of the buffer layer from its $\mathrm{SiC}$ substrate using hydrogenation [40] or after the partial hydrogenation of the graphene itself on top [41, 42]. In both cases a faint band dispersion with apparently linear Dirac cones has been observed. This revealed much more a disordering of the layer than a real "new" semiconducting graphene and, to our knowledge, these seminal results have not solved the question of graphene gap opening by showing transport 
measurements. In the case of functionalization by intercalation, intercalated elements have been observed either between the first ML graphene and the buffer layer, or between the buffer layer and the substrate. As we will see later this strongly depends on the nature of the intercalated atoms. It also depends on the thermal process and layer transformation running during the intercalation, i.e. before, during or after the formation of the graphene layer.

Figure 1 shows a periodic table of elements where the already studied elements are checked. In this quite exhaustive list, we see that many transition metals such as Mn, Fe, Co, Cu, Re, Mo, Ir, Pd, Ni [43, 44, 45, 46, 47, 48, 49] were studied for the realization of electrode with graphene, for their magnetic properties and for spintronic applications. For $\mathrm{Au}[51,50,33]$ and other metals such as $\mathrm{Ag}$ or $\mathrm{Al}[52,33,53,54]$, one of the goals is the search of a good contact material for electronic devices based on graphene. Other elements, such as the ones of Group IV [43, 30, 58, 55, 56, 57, 59, 60] and halogen [61], were also studied. The alkali and the alkaline earth metals (Li, Na, K, Ca, Ba...) were studied because of the analogy with Graphite Intercalation Compounds (GICs) $[62,63]$, with whom applications in energy storage were found [64] and superconductivity was obtained with relatively high superconductivity transition temperatures for $\mathrm{KC}_{8}$ or $\mathrm{Ca}_{6}$ and with critical transition temperatures $T_{C}$ ranging from $<1 \mathrm{~K}$ to $11 \mathrm{~K}[65,66,67]$. Then graphene became a playground to study this property and the role of the graphene sheet in the superconductivity $[68,69,70,71,72,73]$. As superconductivity has been found also for rare-earth metals $\mathrm{GiCs}$ such as $\mathrm{YbC}_{6}$ [65] but not for other rare-earths such as Eu, Cm, Tm [65, 74, 75], they were also studied using graphene [36, 76, 79, 77, 78]. Such elements are well-known for their highly reactive character with carbon atoms and, as we will see afterwards, the intercalation process happens everywhere through the graphene plane without pre-existing defects and even under the buffer layer in the case of graphene/ $\mathrm{SiC}(0001)$. In this system, most of alkali and rare-earth metals are able to intercalate up to the $\mathrm{SiC} /$ buffer layer interface leading to generate a new highly n-doped graphene layer in van der Waals interaction with another graphene plane. This possibly reveals a new class of fermions, the massive Dirac fermions, obtained in van der Waals heterostructures composed of two non-Bravais lattices with a specific stacking and introduced by B. Hunt et al. [80].

Finally all studied elements can be separated into two classes of materials in terms of intercalation, i.e. those for which the intercalation process 

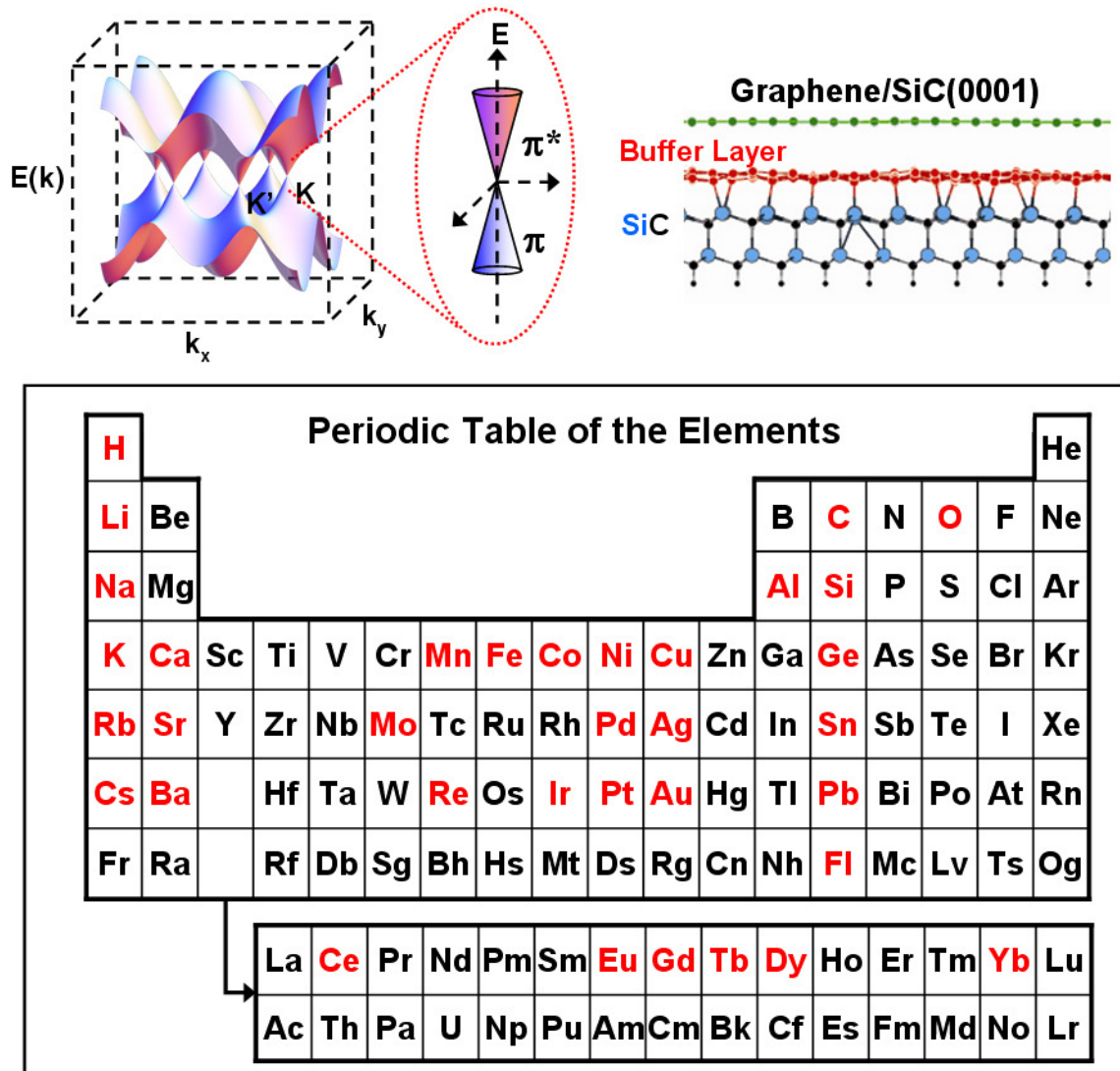

FiguRE 1: Schematic representation of a ML graphene epitaxied on $\operatorname{SiC}(0001)$. The system consists in two graphitic layers. In red is represented the buffer layer which is a carbon layer with a graphene-like arrangement partially covalently bound to the $\mathrm{SiC}$ substrate. The true monolayer graphene (schematized in green) lies on top of the buffer layer in van der Waals interaction. The schematic 3D representation of the graphene band structure with the Dirac cones around the K points of the Brillouin zone is also given. The elements which have been studied on graphene are marked on the periodic table of elements. 
is "difficult" and happens through pre-existing defects, as illustrated by Gold intercalation, and those which are able to react with the graphene plane and intercalate even through several graphene layers by a process of defect creation-intercalation-diffusion with healing during graphene annealing, as exemplary illustrated by Terbium intercalation [36]. In this paper we will first report the results obtained with the intercalation of Gold atoms, particularly on graphene/ $\mathrm{SiC}(0001)$ and the mechanism of intercalation. By comparing with similar other systems, we will describe possible electronic properties, such as spin-orbit (S-O) splitting and their fingerprints in terms of band structure modifications. In a second part, we will report the results obtained with intercalation of rare-earth metals, with an emphasis on Terbium, and the consecutive strong modifications of the graphene band structure. We will show the general character of such band structure modifications and describe the physics behind such as the nature of Dirac fermions and (or) superconductivity. Then we will show that organic molecules may intercalate below graphene, even large ones, opening the way to using graphene as nanocontainer or chemical nanoreactor. Finally, the theme of intercalation will be extended to 2D transition metal dichalcogenides.

\section{Gold intercalation mechanism and electronic effects}

When graphene was still called "monolayer graphite", intercalation of Gold atoms under a graphite monolayer on $\mathrm{Ni}(111)$ substrate was reported, studied by ARPES and High Resolved Energy Electron Loss Spectroscopy (HREELS), leading to the decoupling of the graphene layer from its substrate [81]. Afterwards, the deposition of Gold on graphene was motivated by the possibility to modify the Dirac point position. Indeed, p-type doping was observed with Gold intercalation on graphene/Ni(111) [32] and then on graphene/ $\mathrm{SiC}(0001)[51,31]$. In the aforementioned works [51,31] the deposition of Gold was done on buffer layer and the intercalation at the $\mathrm{SiC} /$ buffer layer interface happened during the annealing of the sample [31]. Working on graphene/ $\mathrm{SiC}(0001)$ too, we have shown in thorough studies that other intercalated Gold structures may be obtained when starting by depositing Gold on a complete graphene topmost layer with subsequent annealing $[50,82,83,84,85]$. The intercalation process is found there to occur between the top monolayer graphene and the buffer layer, and never under the buffer layer. As will be explained, the intercalation happens through the preexisting defects or at the borders between buffer layer and monolayer graphene areas. 


\subsection{Mechanisms of Gold intercalation}

The deposition of Gold was done by evaporation after the complete realization of a monolayer graphene/ $\mathrm{SiC}(0001)$ held at room temperature. It was very difficult to image the graphene sample just after deposition of Gold (i.e. without annealing), as the bonding between Gold and graphene atoms is very weak $[87,88,89]$ and many surface Gold islands were mobile during the scanning process, making the STM tip unstable. Looking for a way to eliminate these Gold islands, we have tested several temperatures of annealing and finally found that cycles of annealing at $1000 \mathrm{~K}$ for $2 \mathrm{~min}$ followed by a decrease of the temperature to $300 \mathrm{~K}$ worked. Indeed, after a few annealing cycles at $1000 \mathrm{~K}$, we obtained a low density of very high and wide 3D islands of Gold on the surface, as seen Fig. 2 (a), following well the predictions based on thermodynamics analysis obtained by DFT calculations of Au on freestanding graphene [89]. Surprisingly, these islands were epitaxied on the graphene surface with a high crystallinity evidenced by the observation of the herringbone reconstruction (see the inset in Fig. 2 (a)), which is usually observed at the surface of perfect $\mathrm{Au}(111)$ single crystal. When we carry on the annealing cycles, the height and width of these Gold islands decreased, their number reduced drastically and we observed the growth of intercalated Au structures [50], as shown in Fig. 2 (b) where a remaining Gold island is pointed out by a blue arrow. As will be explained in the next paragraph, Gold atoms are intercalated between buffer layer and graphene, forming two kind of structures labelled $\mathrm{AuF}$ and IC (ML stands for pristine monolayer graphene). As shown in STM images Figs. 2 (b) to (d), we have observed that all intercalated Gold structures were adjoining large-scale defects, such as puckers, pits (see Fig. 2 (d)) [90] or graphene boundaries (due to the presence of areas of buffer layer or pits). All intercalated Gold structures were surrounding remaining surface Gold islands or were connected via puckers to Gold islands or to a pit containing Gold. We may then infer that these Gold islands are acting as tanks of Gold during the annealing cycles and that the puckers are acting as nanochannels, directing the diffusion of Gold under the graphene layer. Moreover, the preexisting large-scale defects must be involved in the process of Gold atoms intercalation and migration between the graphene layer and the buffer layer. Indeed, similar experiments on metal atoms deposited on graphene have already emphasized the role of large-scale surface defects in the promotion of intercalation, as they provide an access for the diffusing atoms to the surface below the graphene layer [58, 45, 73, 91]. The insertion of foreign atoms between the graphene layer and the buffer layer costs energy, 

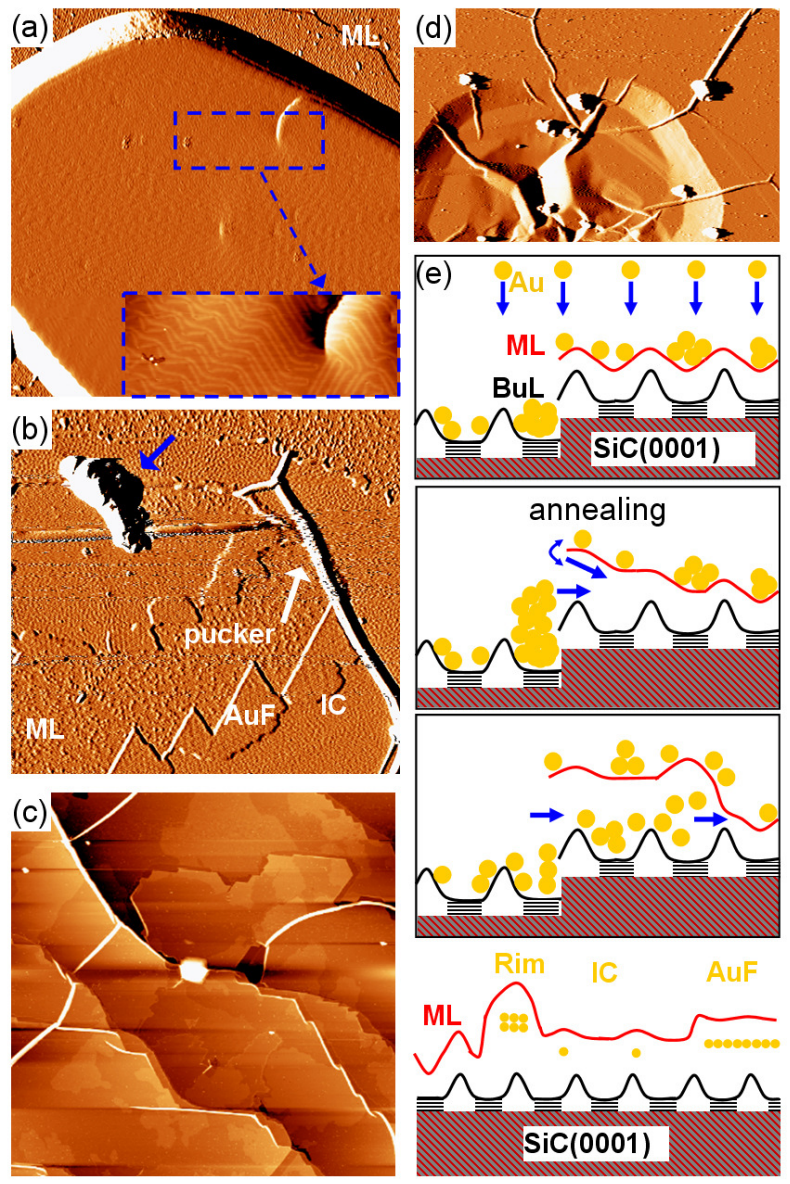

Figure 2: (a) is the STM image of a sample of epitaxial graphene/SiC(0001) after the deposition of $19 \mathrm{ML}$ of $\mathrm{Au}$ and few cycles of annealing at $1000 \mathrm{~K}$ for $2 \mathrm{~min}\left(250 \times 250 \mathrm{~nm}^{2}\right.$, $-1.6 \mathrm{~V}, \partial z / \partial x$-derivative representation of the topography). The inset is a zoom on top of this wide 3D island, showing the herringbone reconstruction of $\mathrm{Au}(111)$ with defects $\left(94.7 \times 35.0 \mathrm{~nm}^{2},-0.3 \mathrm{~V}\right)$. After numerous annealing cycles, Gold is intercalated between the graphene layer and the buffer layer, with scarce and small 3D Gold islands remaining on the surface, as shown by the blue arrow in the STM picture (b) $\left(200 \times 188 \mathrm{~nm}^{2},-1.6 \mathrm{~V}\right.$, $\partial z / \partial x$-derivative representation of the topography). Gold atoms are forming two kind of intercalated structures labelled $\mathrm{AuF}$ and IC, always delimited by an intercalated Gold rim (ML stands for pristine monolayer graphene). (c) The intercalated Gold structures are adjoining puckers, that are forming a network on the surface $\left(600 \times 600 \mathrm{~nm}^{2},-1.5 \mathrm{~V}\right)$. (d) The puckers connect the intercalated Gold structures to remaining surface Gold islands or to pits containing Gold, most probably acting as Gold tanks during annealing cycles $\left(600 \times 384 \mathrm{~nm}^{2},-1.6 \mathrm{~V}, \partial z / \partial x\right.$-derivative representation of the topography). (e) Schemes of the consecutive stages of the so-called defect-aided intercalation process with Gold atoms as yellow dots (see text). (image processing using WSxM software [86]). 
even if the bonding between these two layers is of van der Waals type (the strain energy of dilatation along c axis, although small, still remains). This elastic energy is reduced in the so-called defect-aided intercalation process schematized in Fig. 2 (e). First, the deposited atoms are attracted at the large-scale defects, where the graphene layer is no more continuous. Then, intercalation starts at regions where the graphene layer is released from the buffer layer below, reducing the energetic cost of insertion. For example, the graphene layer may be mobile at its edge during the annealing (vibrational excitation from heating schematized in Fig. 2 (e) by a curved up down arrow ; this is like the bubble mechanism explained in [92]) or a pucker may be formed at the graphene edge during the annealing cycle [93]. Puckers are resulting from the delamination of the graphene layer from the buffer layer. They are probably due to the difference in thermal expansion coefficients of $\mathrm{SiC}$ and of graphene, as the rapid cooling after an annealing causes stress (compressive and/or shear stress [94, 95]) that is released by out of-plane distortions of the graphene layer [93]. They form interconnected networks on the surface, following $\mathrm{SiC}$ step edges or crossing them with no change in size nor direction (see Figs. 2 (c) and (d)). They may be mobile during annealing, carrying along the Gold atoms inside, so that diffusion of Gold atoms in the interlayer space is enhanced. Further diffusion of Gold atoms promotes the intercalation of the remaining surface Gold atoms, as the graphene layer is already apart from the surface and the strain in the graphene layer due to the ripples formed by intercalated structures tends to make it straight.

Moreover, the $\mathrm{SiC}(0001)$ substrate promotes the intercalation process as Gold atoms do not intercalate when deposited on HOPG [96]. Due to their high positive electron affinity and a relatively high first ionization potential, intercalated Gold atoms screen the electrons from the SiC to the graphene layer, as was already proven in Refs. [82, 85]. The energy gained in this process might overcome the elastic energy required to dilate the graphene host, enabling the intercalation and diffusion in the interlayer space $[62,96]$.

\subsection{Gold intercalation : intercalated phases and electronic properties}

After the deposition of Gold and following annealing, we have observed different intercalated phases. Figure 3 resumes the main results obtained on this particular intercalation mode. In Fig. 3 (a) is shown a typical graphene after intercalation of Gold, where pristine monolayer graphene, labelled ML, is easily recognizable by the $(6 \sqrt{3} \times 6 \sqrt{3})$ R30 reconstruction. The area label- 

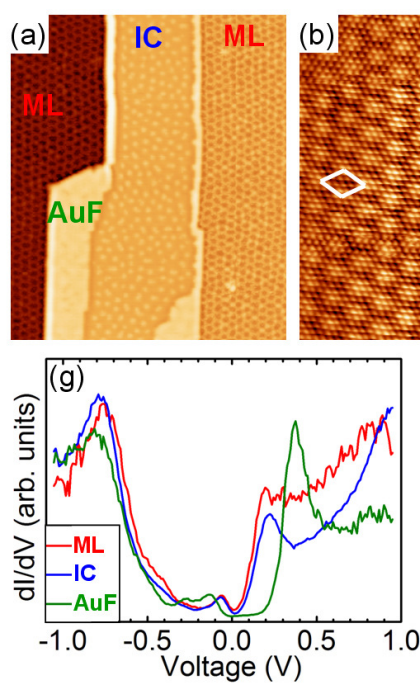

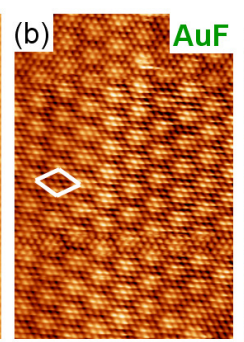

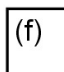
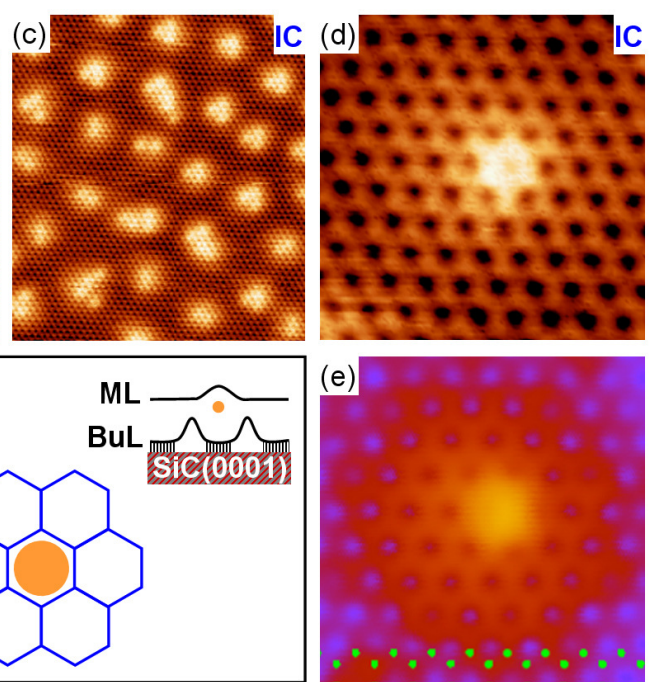

FIgURE 3: (a) is a typical STM image after intercalation of Gold atoms where the two phases co-exist. Pristine monolayer graphene is labelled ML. The area labelled AuF corresponds to the intercalation of a continuous Gold monolayer with the corresponding zoomed image in (b) showing the $(2 \sqrt{3} \times 2 \sqrt{3})$ R30-Au reconstruction indicated by a solid line diamond in white. The diluted phase, labelled IC in (a), is due to the intercalation of single and freestanding Gold atoms with zoomed STM images shown in (c) and (d). (e) is the calculated STM image of a Gold atom intercalated between ML and buffer layer in hollow site, as schematized in fig. $3 \mathrm{f}$ ). (g) shows STS measurements made on ML (in red), on IC (in blue) and on $\mathrm{AuF}$ (in green) domains. (a) : $55 \times 65 \mathrm{~nm}^{2},-1.5 \mathrm{~V}$; (b) : $6.8 \times 10.0 \mathrm{~nm}^{2}$, $-0.2 \mathrm{~V} ;$ (c) : $10.4 \times 12.0 \mathrm{~nm}^{2},-1.6 \mathrm{~V} ;$ (d) $: 2.1 \times 2.1 \mathrm{~nm}^{2},-1.1 \mathrm{~V} ;$ (e) $: 2.1 \times 2.1 \mathrm{~nm}^{2}$, simulated at $-1.3 \mathrm{~V}$. Adapted from [50] and [85]. 
led AuF (for Gold Film) was attributed to the intercalation of a continuous Gold monolayer between buffer layer and graphene [50]. We have evidenced by a fast Fourier Transform (FT) filtering of the STM images (topographic and conductance) several surface reconstructions on AuF domains, like a $13 \times 13-\mathrm{G}$ reconstruction, which has been attributed to a Moiré pattern due to the superposition of the graphene layer on top of a continuous hexagonal $1 \times 1$ Gold monolayer with a lattice parameter of $2.66 \AA$ [50]. In addition, it was observed a $(2 \sqrt{3} \times 2 \sqrt{3})$ R30-Au reconstruction, indicated in Fig. 3 (b) by a white diamond. This surstructure is not a Moiré pattern but is attributed to an electronic effect [50]. Finally, the third observed reconstruction was a $2 \times 2$-Au reconstruction, which is unambiguously a Moiré pattern due to the superposition of the Gold monolayer on top of the underlying $(6 \sqrt{3} \times 6 \sqrt{3})$ R30 reconstruction of the buffer layer. These measurements definitively prove that the Gold monolayer is intercalated between the buffer layer and the top monolayer graphene, preserving the buffer layer.

In the diluted phase labelled IC (for Intercalated Clusters) in Figs. 3 (c) and (d), the bright features were recently attributed to freestanding aggregates of individual intercalated Gold atoms, as the average distance between them was measured $4.17 \AA$ ruling out $\mathrm{Au}$-Au bonds [85]. They were first attributed to triangular clusters of six Gold atoms [82, 84]. However, with the help of calculation, we finally found out that each individual brigth feature was associated to a single Gold atom [85], as shown in Fig. 3 (d), which is a zoom of the STM image of an individual brigth feature. Indeed, the calculated STM image in (e) of a single Gold atom intercalated between the top ML graphene and the buffer layer, as schematized in (f), is in very good agreement with the experimental image (d). Theory ruled out any intercalation under the buffer layer and strongly supported that Gold atoms were freestanding and negatively charged. We tentatively attributed this intriguing repartition of Gold atoms to direct Lennard-Jones and electrostatic (Coulomb, charge-dipole, dipole-dipole) interactions between the aggregates only, without success. We concluded that the interplay between the interactions between Gold atoms and their interactions with the underlying buffer layer was much more complicated [85]. One important result here is that the single Gold atoms are all in hollow position with respect to the top graphene lattice, as schematized in (f) [85]. As explained below, this may have important consequences on the electronic properties particularly for a possible spin-orbit coupling. 

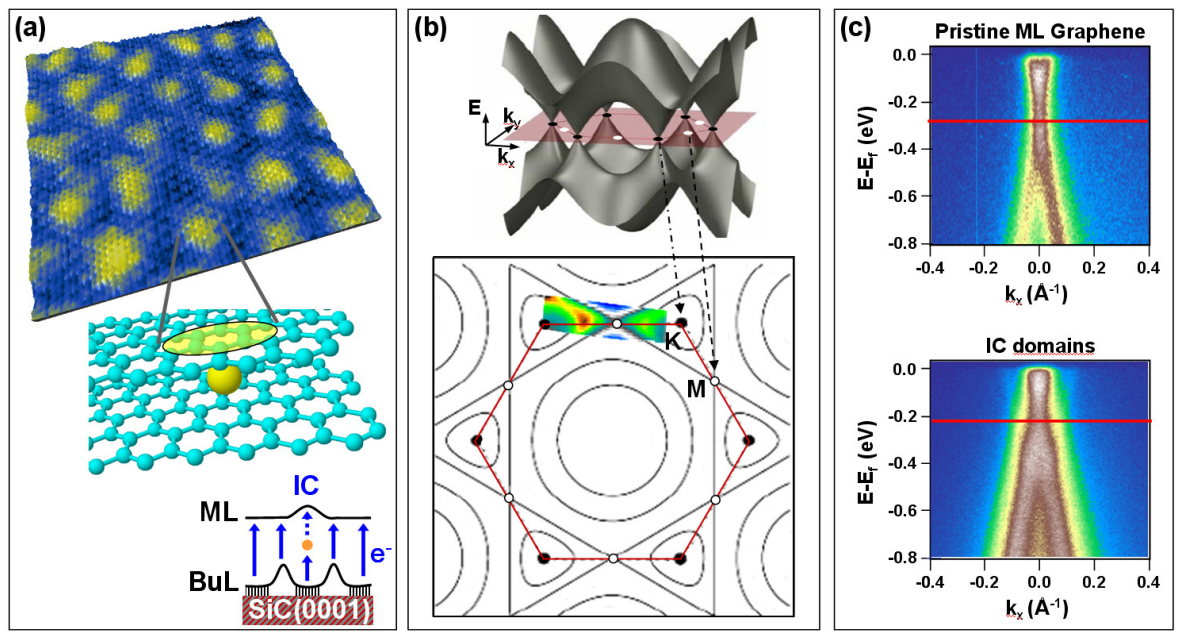

Figure 4: This figure summarizes the effects of the intercalated Gold atoms in the IC phase observed on the top ML graphene. (a) is a 3D topographic STM image (taken at $+\mathbf{8 0 0 m V}$ ) highlighting the feature (in yellow) associated to the Gold atoms under the graphene plane and showing very clearly the standing wave pattern between the features. The partial screening by Gold atoms of electrons transferred from the $\mathrm{SiC}$ substrate is schematized. (b) displays on top a 3D representation of the band structure of graphene with below a 2D constant energy contours (CECs) map calculated by a first-nearestneighbor Tight-Binding approximation. CECs around M points at a binding energy of $-2.3 \mathrm{eV}$ measured by ARPES on IC domains are superimposed on the calculated ones, showing the extension of the Van Hove singularities as predicted by STS measurements in [82]. By comparing detailed ARPES measurements made on pristine ML and on IC domains, one clearly sees in (c) the band structure modifications due to the intercalated Gold adatoms around the Dirac point, i.e. a slight p-type doping and a distortion (swelling) around the Dirac point. This figure is adapted from [82, 84]. 
Fig. 3 (g) shows the superposition of Scanning Tunneling Spectroscopy measurements (STS) done on pristine ML graphene (in red), on AuF domain (in green) and over a brigth feature in the IC domain (in blue). As can be seen, the spectra of pristine ML Graphene and of IC domain are very similar with no change in doping. However, we have proven that the intercalated freestanding Gold atoms partially screened the electron transfer from the $\mathrm{SiC}$ substrate, leading to a framework of standing wave patterns, as shown in figure 4 (a) [82]. These standing waves were the result of an interference process mixing the wave functions associated to the electrons on the graphene plane. By using the Fourier transform scanning tunneling spectroscopy technique (FT-STS), i.e. by performing a FT of the STM conductance images showing the standing wave patterns, it is possible to deduce the mapping of constant energy contours (CECs) at various binding energies, for example to obtain the Fermi surface at the Fermi level [83, 97, 82, 84]. We demonstrated that the new features in the FT images related to the standing wave patterns revealed an increase of the density of states around the M points of the graphene reciprocal lattice leading to an extension of the Van Hove singularities (VHs) [82], which was confirmed two years later by ARPES measurements [84]. The CEC represented Fig. 4 (b) measured at $E-E_{f}=-2.3 \mathrm{eV}$ shows this strong VHs extension caused by the intercalated single Gold atoms. Usually, a strong increase of electron density is related to electron-electron correlation and/or to electron-phonon coupling at the Fermi level. Indeed such a VHs extension and/or trigonal warping effect have been observed by ARPES in the case of heavily n-doped graphene by alkali metals at the Fermi surface [98] and in the case of GiCs [73]. In the case of Gold IC phase, a weak p-type doping of $53 \mathrm{meV}$ was measured. Therefore, this rules out possible electronelectron correlation. Using Tight-Binding theory and modifying in the Dirac equation the hopping potentials of the first, second and even third nearestneighbor carbon atoms, we have found theoretically strong modifications of the Dirac point and even the creation of new Dirac points around the M points, but it was never possible to reproduce such VHs extension [16]. Finally we demonstrated that the local modification of the density of states by only the screening of the electrons on top of an intercalated gold atom is enough efficient to modify drastically the VHs while the electron doping remains very weak!

Another important effect of these freestanding intercalated Gold atoms is the modification of the band structure marked by a swelling around the Dirac 
point $E_{D}$ shown in Fig. 4 (c) [84]. Such band structure modification may be created by different effects. It has been observed in the case of an intercalated Gold monolayer between graphene and a Ni(111) substrate and attributed to a Rahba spin-orbit (SO) splitting [32]. However such a renormalization of the band structure near $E_{D}$ could also be associated to a plasmaron, as first observed in the case of a graphene layer quasi-freestanding on H-saturated $\mathrm{SiC}(0001)$ substrate and weakly $\mathrm{n}$-doped by $\mathrm{K}$ atoms [99]. A plasmaron is a strong coupling between a graphene QP (electron or hole resulting from the photo-emitted electron) and the surrounding plasmons, which are collective electron-electron interactions leading to oscillations of the graphene free electron gas density $[100,101]$. In the aforementioned case of graphene/ $\mathrm{Ni}(111)$ with intercalated Gold atoms, plasmaron as the cause of the renormalization of the band dispersion has been ruled out, as the graphene was undoped with $E_{f}=E_{D}$. It was then unambiguously attributed to Rashba-type SO coupling due to graphene-Gold monolayer interactions, with a large $13 \mathrm{meV}$ SO splitting [32]. A Rashba-SO coupling requires intercalated material with high atomic number and with d states, so that hybridization between these $\mathrm{d}$ states and the $\pi$ states of graphene takes place [102]. For example, no SO coupling was ever observed for monolayers of $\mathrm{Bi}$ (no d states) or $\mathrm{Cu}$ (low Z number) intercalated on graphene/ $\mathrm{Ni}(111)$ [102]. SO coupling was recently observed with a continuous $\mathrm{Pb}$ monolayer intercalated below graphene on a Pt(111) substrate [59] and on a $\operatorname{Re}(0001)$ substrate [60].

Using the same system of graphene/Ni(111) with intercalated Gold, a giant SO splitting of $100 \mathrm{meV}$ has been observed, related to another organization of $\mathrm{Au}$ atoms below graphene [103]. Depending on the deposited quantity of $\mathrm{Au}$ prior to intercalation, Moiré-type $9 \times 9$-Au or $8 \times 8$-Au (with increasing quantity) reconstructions were evidenced by Low Energy Electron Diffraction (LEED), replicas of the $\pi$ band states of graphene being observed by ARPES, but no change in the value of the SO splitting was measured. Based on DFT calculations in GGA approximation and on the fact that the SO splitting was observed to be $\vec{k}$-dependent, it was concluded that this SO splitting is a Rashba-type effect associated to the spin-dependent hybridization of graphene $\pi$ states with $\mathrm{Au} 5 \mathrm{~d}$ states, which were delocalized between 5 to $6 \mathrm{eV}[103,102]$. When the Ni substrate is taken into account in the calculations, the modelized $\mathrm{C} \pi-\mathrm{Au} 5 \mathrm{~d}$ hybridization is shifted from 3 to $4 \mathrm{eV}$ below $E_{f}$ due to Au-Ni hybridization, which was in better agreement with experimental results. It was then experimentally proved that Gold intercalation may induce strong SO splitting. 
The theory showed that the value of SO splitting was drastically dependent on two key parameters : the distance between graphene and the Gold atoms, and the position of $\mathrm{Au}$ atoms with respect to the graphene lattice, i.e. on top or in hollow site. In order to understand the origin of the different values of SO splitting, D. Marchenko et al. [103] modelized three ideal cases (free standing graphene without substrate), presented in Fig. 5. In (a), they considered a monolayer of Gold where every Gold atom is on top of a $\mathrm{C}$ atom in perfect commensuration with the graphene lattice. In this case, they obtained a large SO splitting of $9 \mathrm{meV}$ for an equilibrium distance of $3.3 \AA$. Decreasing this distance increased SO splitting but led to the destruction of Dirac cones, as top position breaks the symmetry between the two graphene sublattices. In (b), they studied the case where each Gold atoms of the previous monolayer was in hollow site. In order to increase $\mathrm{SO}$ splitting whilst preserving Dirac cones, they decreased arbitrary the Gold-graphene distance. They obtained indeed a giant SO splitting with intact Dirac cones but for an energetically unrealistic equilibrium distance. Finally they found that it was possible to obtain by calculation a giant $\mathrm{SO}$ splitting of around $100 \mathrm{meV}$ with intact Dirac cones only in the case where Gold atoms are individual in an ordered structure, in hollow site, with an equilibrium Gold-graphene distance of $2.3 \AA$, as depicted Fig. 5 (c). Coming back to our system, we have obtained the intercalation of a continuous monolayer of Gold but with a lattice parameter which does not lead to a perfect commensuration with the graphene plane [50]. Therefore Gold atoms are in every possible position with respect to the graphene lattice (i.e. top, hollow and intermediates), which is not ideal for a large SO coupling with Gold atoms. However, considering these calculations, IC phase is a very good candidate for a giant SO coupling, as all conditions are gathered : Gold individual atoms, in a quasiperiodic arrangement, all in hollow site with respect to the graphene lattice and with a calculated equilibrium distance of $2.4 \AA$ to ML on top [85]. Moreover, we observed an extension of VHs which coincides with the energy at which hybridization of the $\pi$ band states of graphene with the $5 \mathrm{~d}$ Au states should take place, as seen in Fig. 2 (b) in Ref. [84]. A giant 100meV Rashba-type $\mathrm{SO}$ coupling was already observed on a graphene/ $\mathrm{SiC}(0001)$ sample, which was p-doped due to intercalation of Gold between $\mathrm{SiC}$ and graphene [104]. In this case, it was not clear which kind of intercalated phase was obtained, as they deposited Gold on buffer layer prior to annealing cycles.

Rashba-type SO coupling is interesting for spintronic applications, where 
functionalized graphene may be used for example as electrode for spin polarized current. However, there is completely another physics behind SO coupling, which relates to topological insulator and more generally to Spin Hall (SHE) and Quantum Spin Hall effects (QSHE). QSHE in graphene was first described by C.L. Kane and E.J. Mele [105]. They introduced a new term related to intrinsic SO coupling with specific charasteristic, i.e. the Hamiltonian $H_{S O}$ must preserve the time reversal symmetry and the chirality of the hopping potential around the Dirac point. In other terms, the second-nearestneighbor electron hopping, which consists of an electron hopping between two first nearest neighbors of the same sublattice $\mathrm{A}(\mathrm{B})$, must be spin dependent (pseudospin chirality is preserved). This intrinsic SO coupling generates the opening of a gap at the Dirac points. If the inversion symmetry of the honeycomb lattice is broken, for example due to curvature, to interactions with a substrate or by an applied perpendicular electric field, a competing Rashbatype SO coupling is also induced [1]. The energy gap opening induced by the Kane-Mele SO coupling must be higher than the Rashba-type SO coupling energy, otherwise graphene returns to a zero gap semiconductor with quadratic bands. The theory predicted that QSHE on graphene could be observed but unfortunately, due to the small atomic number $\mathrm{Z}$ of carbon atoms, the induced gap opening is in reality too small for the observation of QSHE at accessible temperature. Six years after the seminal work of C.L. Kane and E.J. Mele [105], C. Weeks et al. [106] theoretically proposed that the decoration of graphene with a dilute concentration of heavy elements could induce an observable energy gap by increasing the robustness of the KaneMele SO coupling and generate a robust topological insulator. As described in the paper, the principle is that an electron of graphene can tunnel onto an adsorbed atom and go back to graphene. In this process, the graphene SO coupling strength is locally enhanced, the QPs remain unchanged, and the band structure of graphene is not modified (the eigen states are not modified by hybridization) but only perturbed with a gap opening. Due to the competition with the Rashba-type SO coupling, that may dominate over Kane-Mele SO coupling, the robustness of the QHSE phase strongly depends on various parameters : the nature of the graphene substrate, the nature of the adatom (for example, it must be nonmagnetic to preserve the time-reversal symmetry [106]), Its position (only a hollow position guarantees to keep the sublattice symmetry intact which preserves the pseudo spin, as first pointed out in Ref. [105]), the type of outer-shell orbitals of the adatom (for example, adatom with partially filled d or f orbitals will induce Rashba-type SO coupling 
which may dominate over Kane-Mele SO coupling [106]) and the coverage of adatoms. Considering all these criteria, Thallium and Indium atoms were supposed to be the best candidates for QSHE observation in graphene [106]. The case of Gold for a robust Kane-Mele SO coupling was the subject of numerous controversy papers. Experimentally, transport measurements made on graphene $/ \mathrm{SiO}_{2}$ decorated by clusters of $\mathrm{Au}$ or Ir adatoms have evidenced no signature of QSHE induced by Kane-Mele SO coupling but rather of a non trivial SHE [107]. S. Abdelouahed et al. [108] studied theoretically the effects of decoration by Gold atoms of graphene at dense coverage considering only the T-type (or top) position (model of free standing graphene with gold asorption without substrate). They found that SO coupling is dominated by Rashba effect, as anticipated by C.L. Kane and E.J. Mele [105]. D. Van Tuan et al. [109] theoretically demonstrated that the clustering of Gold adatoms on graphene has dramatic effects on SHE compared to disordered Gold-decorated graphene. Without going further into this physics, this shows that transport experiments are required to distinguish which contribution (Rashba-type or Kane-Mele SO couplings, SHE, non trivial SHE, etc.) is at the origin of the modifications of the graphene band structure. However, these experiments must be done on very well controlled and characterized samples and all types of gold intercalated phases, especially the IC phase, have to be deeply explored.

\section{Other metals, alkali and rare-earth metals intercalation}

\subsection{Mechanism of intercalation}

Some specific elements, mainly rare-earth metals, some alkali metals and $\mathrm{Al}$, strongly differ from noble metals, exemplified by Gold, as they are able to chemically react with the carbon atoms. During an annealing process, they intercalate homogeneously on the whole graphene surface without pre-existing defects and even under the buffer layer in the case of graphene/ $\mathrm{SiC}(0001)$. The resulting modifications to the graphene band structure are much more pronounced than in the case of Gold, as explained in the next paragraph. To illustrate this assertion, we present the case of Terbium on graphene/ $\mathrm{SiC}(0001)$ [36], shown in figure 6. After deposition of Terbium atoms and a first annealing at $900 \mathrm{~K}$, we observed typical STM images like Fig. 6 (a). The surface was homogeneously covered by distinct Terbium 3D islands with sizes ranging between 20 to $30 \mathrm{~nm}$. These islands were easily picked up by the STM 

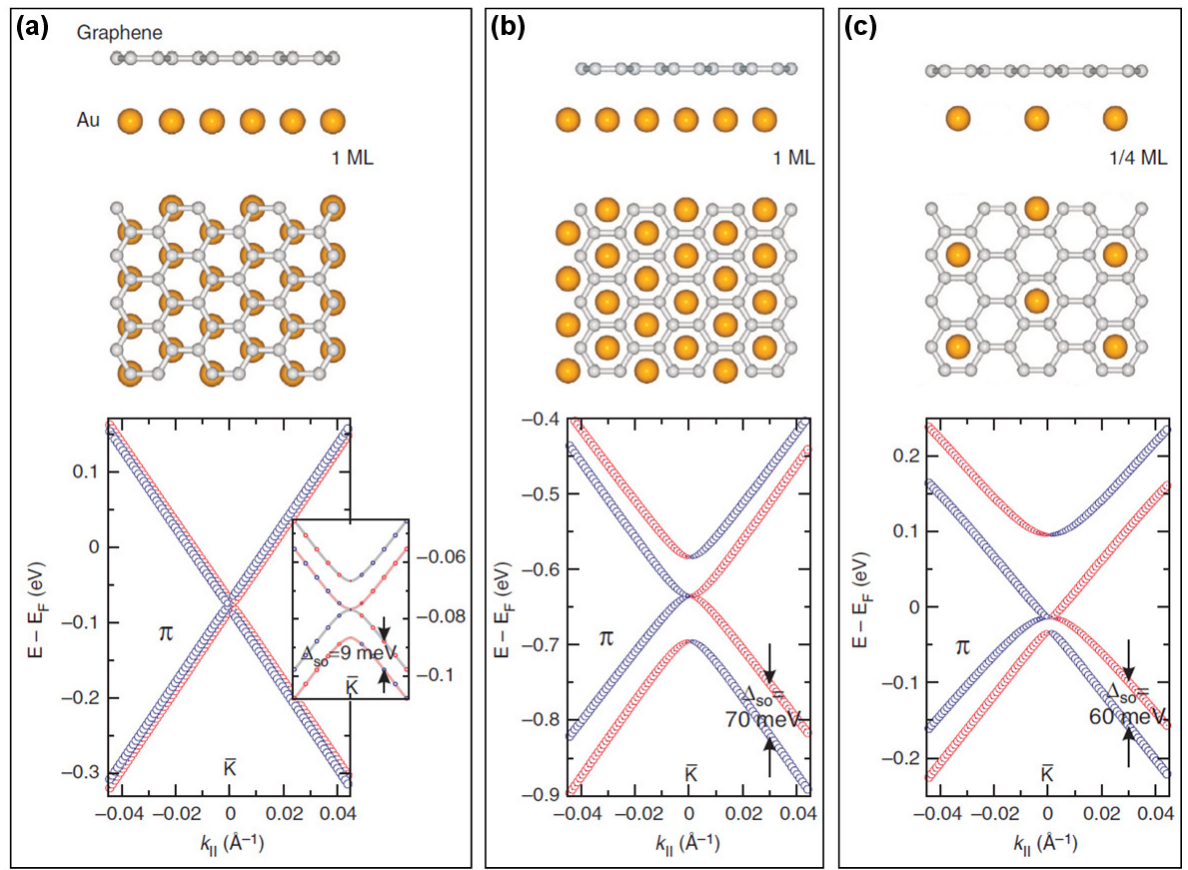

FIGURE 5: This figure presents the three ideal cases of $\mathrm{Au}$ intercalated on graphene/Ni(111) modelized by D. Marchenko et al. [103] and the corresponding effects of the intercalated Gold atoms on the band structure of graphene (see text). This figure is adapted from Ref. [103] with permission. 


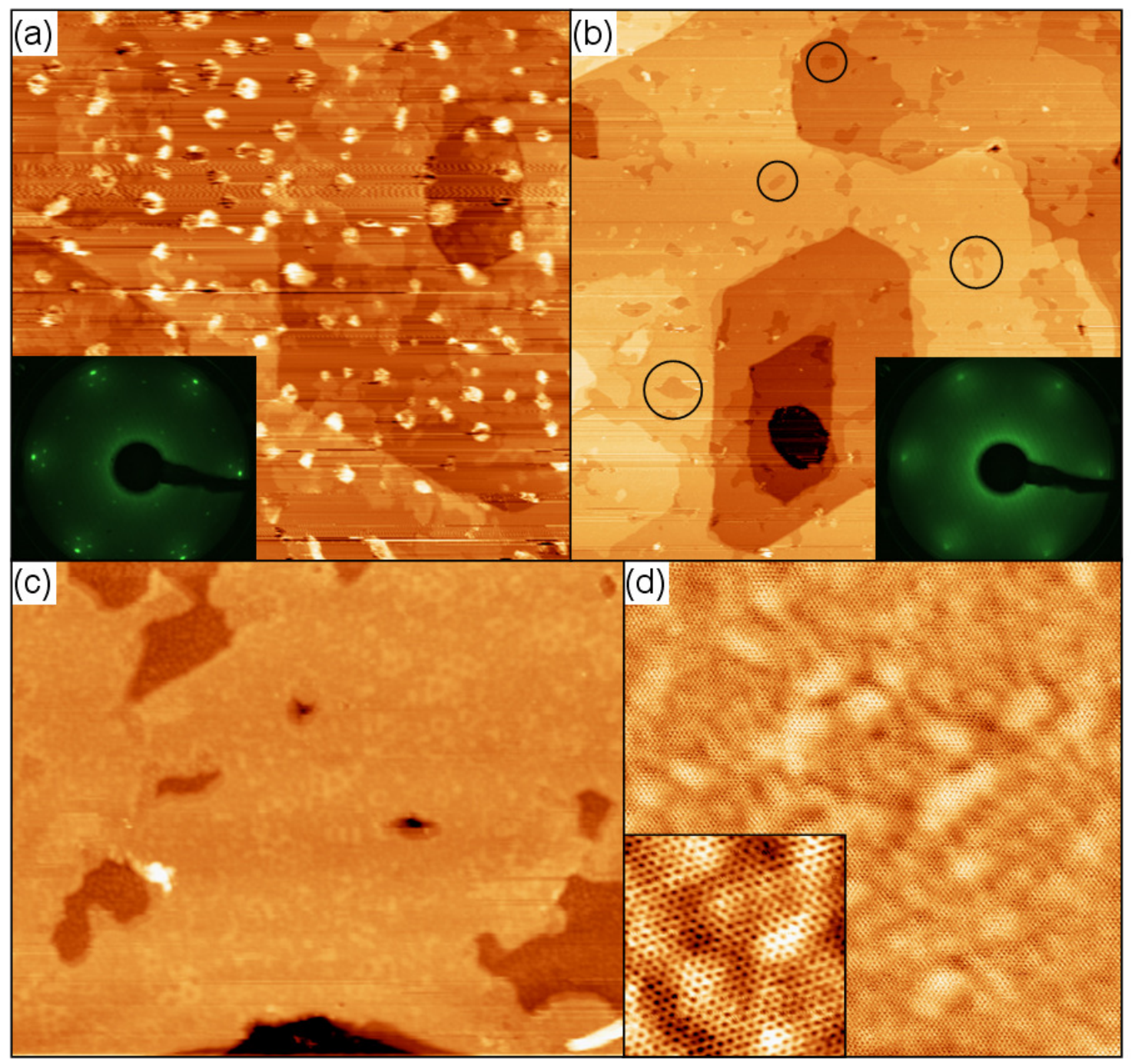

FiguRE 6: STM images and corresponding LEED patterns of Terbium deposited on graphene/SiC(0001). (a) After a moderate annealing at 900K, unstable 3D surface islands of Terbium are homogeneously dispersed on the surface. (b) They disappeared after an annealing at $1100 \mathrm{~K}$ due to the intercalation of Terbium atoms below graphene. The STM image shows small recessed areas (circled), where most probably Terbium atoms intercalate through graphene via the exchange-intercalation mechanism (see text and Fig. 7). The recessed areas are healed with further annealing at $1100 \mathrm{~K}$, as shown in (c). (d) is a zoom-in on a top of a terrace, showing all six carbons of graphene on top of intercalated structures. (a) : $328.1 \times 322.7 \mathrm{~nm}^{2},-2.8 \mathrm{~V}$; (b) : $400 \times 400 \mathrm{~nm}^{2},-1.5 \mathrm{~V}$; (c) : $92.1 \times 75.1 \mathrm{~nm}^{2}$, $-1.9 \mathrm{~V} ;$ (d) $: 25 \times 25 n m^{2},-35 \mathrm{mV}$ and inset : $5 \times 5 \mathrm{~nm}^{2},-90 \mathrm{mV}$. Adapted from [36]. 
tip, explaining the poor quality of the STM images. The brighter spots in the LEED pattern in the inset correspond to the graphene lattice and they are surrounded by the $6 \times 6$-SiC spots related to the buffer layer reconstruction. Turned by $30^{\circ}$ with respect to the $1 \times 1-\mathrm{G}$ spots, the weaker contrasted hexagon with smaller size corresponds to the $\mathrm{SiC}$ lattice. This means that, at this stage, the buffer layer was still intact. No intercalation process was observed and Terbium atoms, which are highly reactive, were oxidized by the residual oxygen contamination in the UHV chamber [36]. After an annealing at higher temperature $(1100 \mathrm{~K})$, the surface Terbium islands disappearead (see Fig. $6(\mathrm{~b})$ ) and there were on the whole surface recessed areas (circled in Fig. 6 (b)) having the same size and distribution as the Terbium surface islands. These recessed areas, covering less than $10 \%$ of the surface, were observed even in the middle of initially flawless terraces of graphene. After a prolonged annealing at $1100 \mathrm{~K}$, the surface covered by the recessed areas was reduced, as in the STM image in Fig. 6 (c). Figure 6 (d) is a STM image on top of a terrace, showing that Terbium atoms were intercalated under the graphene layer without apparent ordering and that this graphene layer showed no atomic-scale defects. Moreover, all $\mathrm{C}$ atoms of the graphene lattice were imaged at low bias (see the inset) whereas the underlying reconstruction of the buffer layer was no more visible. Indeed, the LEED pattern in Fig. 6 (b) showed the graphene lattice spots, but all other features attributed to the buffer layer and the $\mathrm{SiC}$ substrate have disappeared. Terbium atoms, by intercalating until under the buffer layer, were decoupling it completely from the $\mathrm{SiC}$ substrate [36]. As Terbium atoms are highly reactive and as the intercalation happened homogeneously everywhere on the whole surface, we may infer that Terbium intercalation on graphene/SiC $(0001)$ proceeded like the so-called exchange-intercalation mechanism that was assumed for intercalation of several reactive species on graphene/metal single crystals, as depicted in Fig. 7 [44, 110, 91]. After deposition of reactive species, intercalation proceeds in transient stages, the first one being the breaking of $\mathrm{C}-\mathrm{C}$ bond and the bonding to the intercalant atoms on top (the probability increases with the temperature of annealing and the reactive character of the intercalant) leading to the generation of atomic-sale defects. The intercalant atoms diffuse then through these defects and form an intercalated structure. The last step is the self-healing of the graphene layer on top, if the annealing temperature is high enough. A similar intercalation mechanism has been recently observed in the case of sub-monolayer growth of Titanium, Cobalt, Palladium [111] and Manganese atoms [45] on graphene/SiC(0001). 


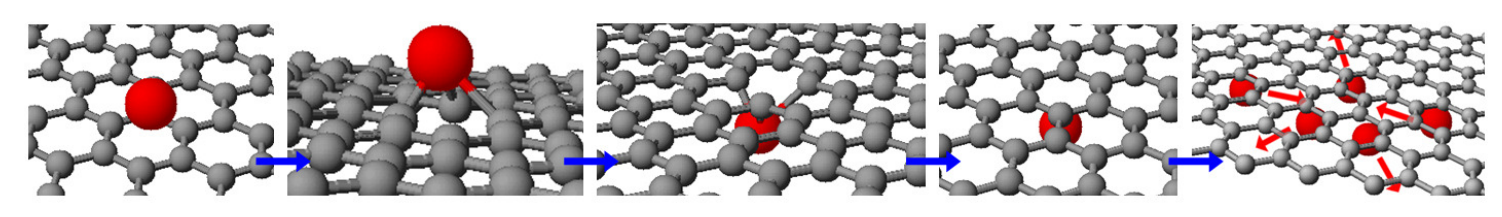

FiguRE 7: Schemes presenting the consecutive stages of the so-called exchangeintercalation mechanism assumed for the intercalation of Terbium atoms (in red) below graphene (in grey). After the deposition on a perfect graphene layer, Terbium atom form a transient covalent bonds with $\mathrm{C}$ atoms during annealing. Then it diffuses through this atomis-scale defect. While continuing annealing, graphene heals itself and intercalated Terbium atoms diffuse in the interlayer space and form an intercalated phase.

The most interesting case is the one of Titanium : Ti atoms were forming 3D nanoislands on graphene/ $\mathrm{SiC}(0001)$ after annealing at $400 \mathrm{~K}$. While scanning, the STM tip removed the islands, leaving extended holes with damaged graphene, due to the dissolution of the graphene layer in contact with the Ti surface island [111]. The sample was not further annealed. By comparison with the Terbium experiments, we may suppose that the recessed areas seen in Figs. 6 (b) and (c) are obviously the entrance position of Tb atoms during the intercalation process and that they are self-healed during subsequent annealing. Of course, annealing at high temperature may induce a competition between the intercalation-atomic diffusion processes and the desorption of intercalant atoms. Recently, by studying the intercalation of Europium atoms on graphene/SiC(0001), S. Sung et al. [76] found that the intercalation between graphene layer and buffer layer started already at RT up to $400 \mathrm{~K}$, temperatures much lower than those usually used in the scientific literature including us (beyond 900K). Annealing at $600 \mathrm{~K}$ led to a deeper intercalation of Eu atoms below buffer layer with the formation of Eu-Si bonds [76]. A higher annealing temperature led to a clustering phase of metallic $\mathrm{Eu}(2.5 \mathrm{~nm}$ diameter) randomly distributed at the vertices of the $6 \times 6$-SiC reconstruction of the buffer layer [112]. Desorption of intercalated Eu atoms started at $1320 \mathrm{~K}[76,112]$. These results demonstrate the possibility to accurately control the intercalation process allowing the realization of complex van der Waals 2D heterostructures.

\subsection{Electronic properties}

\subsubsection{Mixed massive and massless fermion}

In the previous paragraph we have shown that some specific atoms were highly reactive with graphene and were then able to intercalate directly 


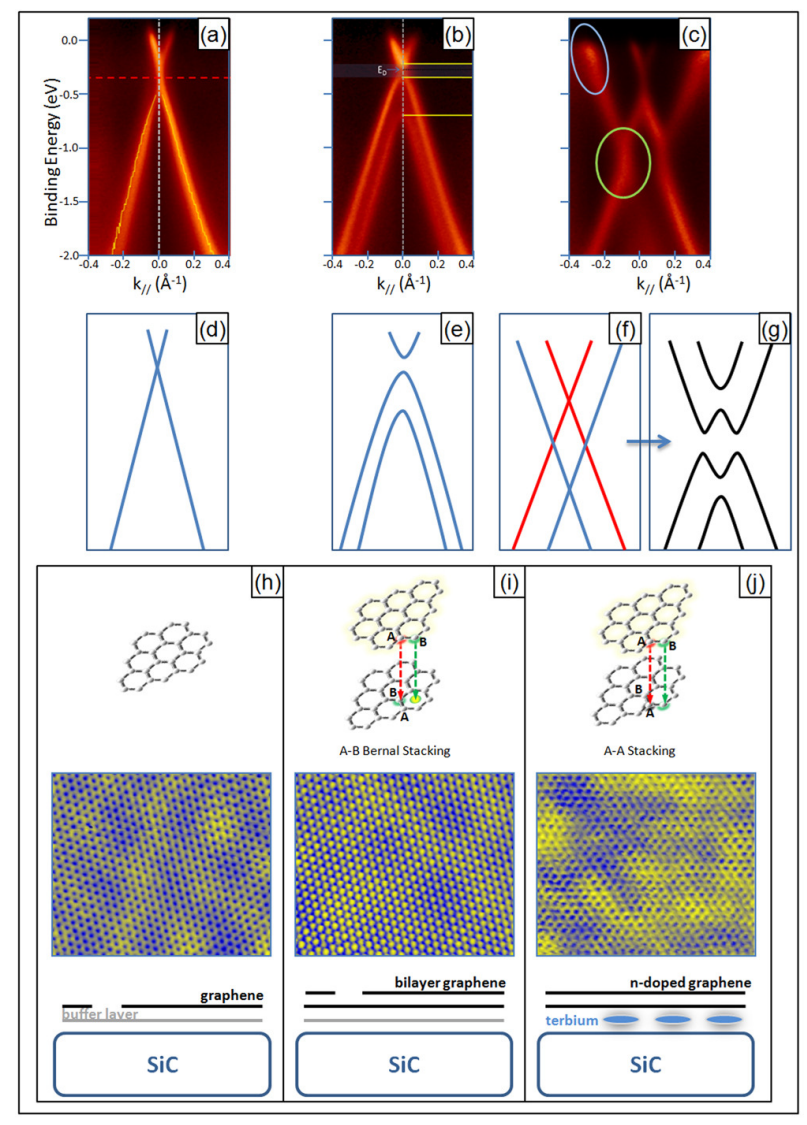

FIGURE 8: (a) to (c) ARPES measurements around $\mathrm{K}$ point respectively for a monolayer graphene/ $\mathrm{SiC}(0001)$, for a bilayer graphene/ $\mathrm{SiC}(0001)$ and for a monolayer graphene/SiC(0001) after intercalation of Terbium atoms (adapted from [36]). In (d) to (f) the resulting band structures are schematized. (h) to (j) present the corresponding schematic representations of the system with the corresponding STM images at low bias. (g) corresponds to a schematic interpretation of the band structure in (c), following the interpretation of K.S. Kim et al. [113]. As explained in the text, such complex band structure may be the result of the mixing in the same system of massive and massless Dirac fermions due to the A-A stacking with a slight twist of two graphene layers not equivalently doped. 
through several graphene layers. In this case, the measured band structure (for example in the case of graphene/ $\mathrm{SiC}(0001)$ or $/ \mathrm{Ni}(111)$ ) presented systematically drastic modifications with respect to the initial dispersion of pristine graphene layer, as shown in figure 8 (c). Figure 8 consists in three columns presenting characteristics of monolayer graphene (left column), of bilayer graphene in Bernal stacking (middle column) and of a graphene layer with intercalated elements (right column).

For monolayer graphene, an electron hopping from one atomic site to its first nearest neighbor does not "feel" the atomic lattice (second and third neighbors) at low energy around the $\mathrm{K}$ points. Thus, the effective mass of the QP tends to zero, therefore QP are called massless fermions. This leads to the apparition of Dirac cones around $\mathrm{K}$ points where the $E(k)$ dispersion is linear, as observed by ARPES in Fig. 8 (a) and schematized in Figure 8 (d). The measurements in (a) were done on graphene/ $\mathrm{SiC}(0001)$, with $E_{D}$ located at $0.45 \mathrm{eV}$ below the Fermi level due to n-type doping from $\mathrm{SiC}$ substrate. STM imaging at low bias on this system shows all six carbon atoms of the graphene honeycomb (see Fig. $8(\mathrm{~h})$ ) and the underlying $6 \times 6$-SiC reconstruction related to the partial bonding of the buffer layer to $\mathrm{SiC}$ substrate. In the case of bilayer graphene, the Bernal stacking of two graphene layers, depicted in Fig. 8 (i), leads to the overlapping of the $\pi$ band states of every second carbon atoms which allows the hopping of electron from one graphene layer to the other. Then the electrons of one graphene layer "feel" the atomic lattice of the second layer (similar to a pseudopotential) and acquire an effective mass, therefore QP are called massive Dirac fermions. As a consequence, the band dispersion is no more linear but parabolic, turning the Dirac point into a small gap, as measured by ARPES on bilayer graphene/SiC(0001) in Fig. 8 (b) and schematized in Fig. 8 (e). STM imaging at low bias on bilayer graphene shows carbon atoms of only one graphene sublattice, with a fainter $6 \times 6$-SiC reconstruction (see Fig. 8 (i)).

In the case of bilayer graphene in A-A stacking, as depicted in figure $8(\mathrm{j})$, the standard Tight-Binding theory predicts two interpenetrating Dirac cones merging into a Dirac circle at $E_{D}$. The linear band dispersions are shifted by an energy of $2 \gamma^{\prime}$, where $\gamma^{\prime}$ is the interlayer hopping energy [114, 115], as depicted in Fig. 8 (f). This energy shift should depend of course on the substrate and on an interlayer field if the graphene layers are not equivalently doped. K.S. Kim et al. [113] studied the effects of weak doping by decoration of Potassium atoms on top of a quasi freestanding bilayer graphene. Starting with a monolayer graphene/SiC(0001) where the buffer layer was decoupled 
from the substrate by hydrogenation, the sharp ARPES measurements revealed a band structure with two parabolic band dispersions and a slight p-doping, as the inital Dirac point at $0.45 \mathrm{eV}$ below $E_{f}$ was shifted to $0.15 \mathrm{eV}$ above $E_{f}$. By depositing Potassium atoms on top, they increased step by step the n-doping of the topmost graphene layer and they followed the emergence of two linear bands leading to a band structure similar to the one in figure 8 (c). They interpreted the two linear shifted cones as a breaking of the lattice symmetry leading to two monolayers in A-A stacking, corresponding to the band structure schematized in Fig. 8 (f). Studying very precisely the intensity of the expected circular contour around $E_{D}$ in $\left(k_{x}, k_{y}\right)$ constant energy map, they evidenced a Dirac arc structure with a high intensity anisotropy along the contour. They concluded that there was a slight misorientation of $\sim 0.1^{\circ}$ between the two graphene layers, leading to the transformation of the band structure from figure 8 (f) (pure massless fermions) to (g) (mixed massless and massive fermions). They also observed an exotic chiral pseudospin texture. If we compare our band structure shown in Fig. 8 (c) to the one obtained by K.S. Kim et al. [113], we may expect the same kind of behavior when the quasi-freestanding bilayer graphene is obtained by the intercalation of atoms between the buffer layer and the $\mathrm{SiC}$ substrate, leading to two graphene layers not equivalently doped. Indeed, the band structure shown in Fig. 8 (c) was obtained after the intercalation under the buffer layer at 1100K of Terbium atoms on a monolayer graphene/SiC(0001), as presented in the previous section. As explained in [36], one Dirac cone corresponded to a highly n-doped monolayer graphene with a Dirac point $E_{D 1}$ at $1.59 \mathrm{eV}$ below the Fermi level, and the other Dirac cone had its Dirac point $E_{D 2}$ unchanged compared to pristine monolayer graphene, as shown Fig. 8 (a). The highly n-doped monolayer graphene was created by the decoupling of the buffer layer from its substrate and this led to a sample made of two stacked graphene layers with a pronounced difference in doping level. Moreover, our STM images shown in Fig. 8 (j) strongly support a A-A stacking, as the six carbon atoms were visible at low bias voltage whereas the buffer layer reconstruction was no longer observed. There should be also a very weak misorientation (less than $1^{\circ}$ ) since only one Brillouin zone was visible in LEED patterns and no Moiré patterns were observed by STM at larger scale.

In the case of functionalized graphene, the intercalation of various elements leads to the same complex band structure, for example in the case of graphene/SiC(0001) : Rubidium and Cesium [72, 73, 79, 116], Ytterbium [77], Lithium [70, 117], Sodium [118], Cerium [78], Aluminium [54] and Europium 
$[79,76]$. Such complex band structure has been observed too with other systems, for example in the case of a kagome lattice of Fe atoms superimposed on a 2D stanene monolayer at the surface of a $\mathrm{Fe}_{3} \mathrm{Sn}_{2}$ monocristal [119] but also probably in the case of $\mathrm{Ca}_{6}$ graphite intercalation compound [120]. This suggests that this kind of fermiology is probably much more general and worth investigating in detail. Moreover, such systems may find applications in " $2 \mathrm{D}$ valleytronics".

\subsubsection{Intercalation and superconductivity}

As already mentioned in this article, the intercalation studies of graphene were first motivated by the seminal works done on GICs and the observation of superconductivity [62]. In the case of GICs, the superconductivity mechanism and the interplay between the intercalant and the stacking of graphene planes were not fully understood. The question was raised whether the superconductivity was parallel to the graphene plane in the host graphite structure (i.e. within individual graphene plane) or perpendicular (i.e. involved in several planes). This is why graphene became a playground to study superconductivity as it offers the possibility to control precisely the number of graphene layers, their stacking orientation and to play with the graphene/substrate interaction by changing the substrate. There are different types of superconductivity mechanism. In the case of GICs, the superconductivity mainly observed with alkali metals is described by the model of J. Bardeen, L. Cooper and J. Schrieffer (BCS) [120]. This may be also the case of graphene functionalized by intercalation but other possible mechanisms have been mentioned, as explained later.

In the microscopic description of the BCS superconductivity mechanism, the electrons are paired at the Fermi level by the interaction with the phonon lattice, which overcomes the electrostatic repulsion between electrons. The electrons are paired by an energy corresponding to a phonon vibration mode $\hbar \omega_{p}$ and the strength of the electron-phonon coupling (EPC) is $\lambda$. The superconducting critical temperature $T_{c}$ is usually estimated in the case of $\mathrm{GiCs}$ using the following McMillan formula :

$$
k_{B} T_{C}=\frac{\hbar \omega_{p}}{1.45} \exp \left(-\frac{1.04(1+\lambda)}{\lambda-(0.62 \lambda+1) \mu *}\right)
$$

with $\mu *$ the effective Coulomb repulsion energy. Thus $T_{c}$ is theoretically directly proportional to the phonon energy and increases with $\lambda$. Both can 
be deduced from ARPES measurements, particularly in the case of graphene which shows a simple Dirac cone dispersion : $\lambda$ is deduced from the curvature of the kink corresponding to the real part of the spectral function, and $\hbar \omega_{p}$ from the broadening of the band much more related to the imaginary part. Both are however linked via a function called the Eliashberg function, which may be extracted from ARPES data using a detailled analysis of the momentum dispersion curves (MDC). A.V. Fedorov et al. [121] systematically studied the link between EPC strength $\lambda$ and the electron density doping. In their experiment, they used monolayer graphene/ $\mathrm{Ni}(111)$, where graphene has been decoupled from the Ni substrate by intercalation of Au. They have then evaporated alkali and alkaline earth metals ( $\mathrm{Cs}, \mathrm{Rb}, \mathrm{K}, \mathrm{Na}, \mathrm{Li}$ and $\mathrm{Ca}$ ) and extracted systematically the EPC $\lambda$ in function of the electron density. They found $\lambda=10^{-9} \sqrt{D}$ with $\mathrm{D}$ the density of electrons. They estimated $T_{c}$ based on the extracted $\lambda$ using the McMillan formula but it was not possible to measure it directly, as explained later.

This study shows discrepancies with the superconducting GICs. For example, in the case of GICs, the dependence of $\lambda$ with the electron density is linear [120]. The link between $\lambda$ and superconductivity, and more precisely the determination of $T_{c}$ has not been systematically done in the case of graphene. For GICs, $T_{c}$ can be measured without contacting the sample. It is determined by the paramagnetism transition temperature using Superconducting Quantum Interference Device (SQUID) magnetometry measurements. In this case, the link between $\lambda, T_{c}$ and the transferred electron density (n-doping density) has been experimentally established. In a systematic study, T. Valla et al. [122] compared the non-superconductive GICs $\mathrm{KC}_{24}, \mathrm{Li}_{6}$ with the superconductive $\mathrm{KC}_{8}$ (with $T_{c}=0.5 \mathrm{~K}$ ) and $\mathrm{Ca}_{6}$ (with $T_{c}=11 \mathrm{~K}$ ). They found that the experimental value of $T_{c}$ fits very well its theoretical value using the McMillan equation and the measured $\lambda$. They also found that $\lambda$ increases with the transferred electron density and that a minimum of electron density is required (typically $10^{13} \mathrm{~cm}^{-2}$ ) with a $\lambda$ of 0.6 in the case of $\mathrm{CaC}_{6}$. They also established clearly that both graphene-derived electrons and graphenederived phonons are involved in the superconductivity of GICs. In the case of graphene, for which the SQUID magnetometry signal is too small, $T_{c}$ can be determined only by direct transport measurements. This requires to make contacts having a resistivity varying sufficiently monotonically with the temperature in order to be able to follow the superconductivity transition temperature. This explains why $T_{c}$ has been more rarely (and never systematically) determined in the case of graphene and why researchers first 
focused much more on $\lambda$. In the case of graphene, typical values of $\lambda$ were lower than for GICs [121]. The highest was determined for $\mathrm{Ca}_{6}$. They found 0.16 for an electron density D of $5.10^{14} \mathrm{~cm}^{-2}$ and S. Ichinokura et al. [71] measured directly a $T_{c}$ of $4 \mathrm{~K}$ using a four-probe STM.

In the previous description of superconductivity the electrons are paired via electron-phonon coupling. However there are other possibilities to pair carriers leading superconductivity. They are classified by the momentum dependence of their superconductive gap, i.e. if there is (or not) an anisotropic superconductive band gap depending on the directions in the reciprocal Brillouin zone and belonging to which symmetry group. In the case of BCS theory, there is no momentum dependence of the superconductive gap leading to the so-called conventional s-wave superconductivity. There are other types of symmetry pairing where the electron with specific momentum are paired, as in the case of d-wave superconductivity predominantly observed for high $T_{C}$ cuprate superconductors [123]. In this case the pairing symmetry is due to the nesting of the density of states at the Fermi level. The density of states, which is proportional to $\nabla_{k}^{-1} E(k)$, diverges when the Fermi level is at an energy corresponding to a flat-band portion (quasi non-dispersive band) of the constant energy contour (CEC). This can happen at specific positions in the first Brillouin zone, as exemplary experimentally illustrated by K. McElroy et al. [124]. They studied by FTSTS the QPs interference processes observed at the Fermi level on the surface of a clived superconduc-

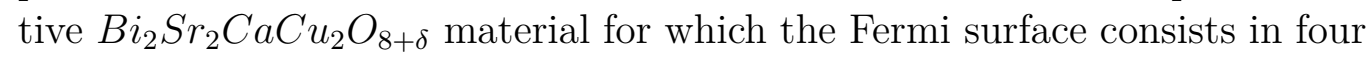
banana-shaped CECs with a strong increase of the density of states at the apex of each banana. In the case of a 2D material with non-Bravais lattice such as graphene, the band structure has a similar nesting effect around the so-called Van Hove singularities (VHs) at the M points, as already discussed in the case of Gold intercalation and schematized on the band structure figure 4 (b). In this first highly controversial VHs scenario [125], superconductivity could be observed if the Fermi level is positioned at the energy corresponding to the VHs. Twenty years later, R. Nandkishore et al. [126] theoretically predicted for the specific case of graphene a possible "chiral superconductivity" where the electrons are paired for momentum pointing the hexagonal six VHs (in the framework of this paper we will not discuss in detail the "chiral" aspect which is due to a breaking of time reversal symmetry). These prediction is of course relevant for other 2D systems with non-Bravais lattice similar to graphene. This is why, as discussed in the case of Gold and rare-earth metals intercalation, numerous studies were devoted to obtain by 
intercalation highly n-doped graphene in order to align the Fermi level with the VHs position. It is also possible to move the VHs by twisting two layers of graphene. This has been shown experimentally by G. Li et al. [127]. The twist of two graphene layers generates a Moiré pattern which creates different types of stacking between the sublattices A and B of each graphene layer. S. Fang and E. Kaxiras [128] found theoretically that at a magic angle of $1.06^{\circ}$ the band structure presents a flat band at the Fermi level located over A-A stacking sites forming a Moiré pattern. Very recently in two papers published in Nature, Y. Cao et al. used their developped 'stear and stack' techniques to produce van der Waals heterostructures with controled twist angle between two stacked graphene planes. They performed magnetotransport measurements and showed experimentally the emergence of these flat bands at the predicted magic angle [129]. In the second article they found a superconductive transition at 1.7 [130] reporting the first observation of unconventional superconductivity using only graphene sheets.

In this research field also, intercalation offers another degree of freedom to modify and control a van der Waals heterostructure. Considering the interplay with the possible modifications of stacking sequences between two graphene layers with intercalated atoms, as discussed in the case of massivemassless Dirac fermions, these last publications demonstrate how large is the field of possibilities offered by intercalation.

\section{Intercalation of molecules}

The intercalation process on graphene does not take place only with atomic species but also with molecules, even large ones. Extensive studies about intercalation below graphene of molecules such as Hydrogen, Oxygen, Carbon Monoxide water were conducted, for example in order to know if graphene may be used as a coating protecting the underlying substrate against oxidation.

Oxygen intercalation on graphene epitaxied on weakly (e.g. Pt, Cu) as well as strongly (e.g. Ni, Ru) interacting metal substrates has been investigated by a number of studies. In the case of the strongly interacting substrate $\mathrm{Ru}(0001)$, an initial study by H. Zhang et al. [131], where the authors used STM and XPS techniques, reported the intercalation of Oxygen molecules at graphene $/ \mathrm{Ru}(0001)$ interface after annealing at $600 \mathrm{~K}$. A number of other studies also suggest that the oxygen intercalation at graphene $/ \mathrm{Ru}(0001)$ interface can only be thermally activated due to the high interaction of graphene 
with $\operatorname{Ru}(0001)$ susbtrate $[132,131,133,134,135]$. Recently E. Gränäs et al. [136] using STM and XPS demonstrated that monolayer graphene/Ir(111) is impermeable to $\mathrm{O}_{2}$ even using a high $\mathrm{O}_{2}$ pressure of $10^{-3} \mathrm{mbar}$ with a temperature up to $700 \mathrm{~K}$, however Oxygen intercalation can take place at $10^{-5} \mathrm{mbar}$ $\mathrm{O}_{2}$ pressure and $410 \mathrm{~K}$ for 0.5 monolayer of graphene/ $\operatorname{Ir}(111)$. This means that graphene edges promote the intercalation of Oxygen below graphene. R.S. Weatherup et al. [137] observed that for weakly interacting substrates like $\mathrm{Pt}(111), 2$ days exposure to air in ambient conditions led to intercalation of Oxygen on monolayer graphene/Pt(111) resulting in freestanding graphene, whereas monolayer graphene on strongly interacting substrate $\mathrm{Ni}(111)$ showed no sign of intercalation. However intercalation of Oxygen on monolayer graphene/Ni(111) at $470 \mathrm{~K}$ started through defects of graphene domains rotated with respect to $\mathrm{Ni}(111)$, as suggested in a very recent study by $\mathrm{L}$. Bignardi et al. [138]. Since it is established by several studies that intercalation of $\mathrm{O}_{2}$ is more difficult for a complete monolayer coverage of graphene compared to graphene flakes, it can be concluded that graphene edges are more favorable for Oxygen intercalation than graphene defects, such as point defects or domain boundaries. In the case of Carbon Monoxide, its intercalation under graphene was studied with various metal substrates, such as the strongly interacting $\mathrm{Ru}(0001)$ [139] and $\mathrm{Ni}(111)$ [140] and the weakly interacting Pt(111) [141, 142] substrates. Using STM, photoemission spectroscopy (XPS, UPS), $\mu$-LEED and low-energy electron microscopy (LEEM), L. Jin et al. [139] showed that exposure at ambient pressure to CO molecules of submonolayer as well as monolayer graphene $/ \mathrm{Ru}(0001)$ resulted in a homogeneous intercalation of $\mathrm{CO}$ molecules, with ordered $\mathrm{CO}$ molecules at graphene-Ru interface. The authors carried out extensive XPS study at different pressure of $\mathrm{CO}$ molecules and suggested that $\mathrm{CO}$ intercalation started to significantly take place at $13 \mathrm{mbar} \mathrm{CO}$ at room temperature. E. Gränäs et al. [143] demonstrated CO intercalation at graphene/Ir(111) interface at room temperature using CO pressure close to 1mbar. LEED and STM showed a $(3 \sqrt{3} \times 3 \sqrt{3}) R 30$ superstructure formed by intercalated clusters of 19 $\mathrm{CO}$ molecules, which is similar to the adsorption structure formed on clean Ir(111) upon mbar CO exposure. CO partial pressure required for the intercalation below graphene grown on different substrates seems to depend mostly on the bonding strength between graphene and its substrate, as suggested by the currently available experimental data [139]. Few studies have been made on the intercalation of water below graphene and they tend to show that intercalation does not depend in this case on the graphene-substrate bonding 
strength. Indeed, X. Feng et al. [144] have made a comparative study about the intercalation of $\mathrm{H}_{2} \mathrm{O}$ molecules on graphene epitaxied on $\mathrm{Ru}(0001)$ and $\mathrm{Cu}(111)$. Using STM, they showed that water molecules can intercalate below graphene $/ \mathrm{Ru}(0001)$ at temperatures as low as $90 \mathrm{~K}$ due to the presence of line defects (dislocations and grain boundaries) in the graphene layer. Indeed, right after deposition water molecules accumulated along these defects and after annealing, seemed to dissociate and break the $\mathrm{C}-\mathrm{C}$ bonds at theses line defects, inducing the fragmentation of the graphene layer which is followed by intercalation of $\mathrm{H}_{2} \mathrm{O}$ below those graphene flakes. In the case of graphene/ $\mathrm{Cu}(111)$, once more water molecules were observed to accumulate at line defects but annealing at $150 \mathrm{~K}$ led to occasional splitting up of the graphene layer followed by intercalation below the few graphene flakes. This indicates that the underlying substrate plays an important role in determining the chemical reactivity of the graphene layer, as substrate may promote to catalyze water dissociation on graphene.

Surprisingly even large molecules are able to readily intercalate below graphene layer. For example, M. Ohtomo et al. [145] have reported intercalation at room temperature of 1-octanethiol molecules between graphene and a $\mathrm{Cu}$ foil substrate after immersing the samples. Intercalation happened through graphene defects such as grain boundaries and led to the formation of stable self-assembled monolayers due to covalent bonds of thiol molecules with $\mathrm{Cu}$. Larger molecules, such as $C_{60}$ and $C_{70}$ intercalate below graphene too. Based on Auger Spectrocopy measurements, E.V. Rut'kov et al. [146] showed that deposited $C_{60}$ molecules were able to intercalate under a monolayer graphene/Ir(111) after annealing at 800K. A. Varykhalov et al. [147] reported the intercalation of $C_{60}$ molecules under graphene/Ni(111) after annealing at 700K. STM images at different bias conditions showed surface corrugations which were unambiguishly attributed to change in local density of states due to intercalation of $C_{60}$ molecules. The authors showed the potential of STM to visualize the intercalated molecules under graphene. E. Monazami et al. [148] have reported a method to control the local strain in graphene on $\mathrm{Cu}(100)$ by intercalation of $C_{60}$ molecules. Authors have proven intercalation by comparing STS spectra obtained on intercalated regions with those made on bare graphene/ $\mathrm{Cu}(100)$ and those made on $C_{60}$ molecules residing on the surface. An approach towards controlled intercalation of $C_{60}$ molecules was also discussed which may lead to formation of a crystalline lattice of $C_{60}$ molecules and therefore may give control over strain in graphene lattice.

In the case of molecular intercalation below graphene grown on non-metallic 
substrates such as $6 \mathrm{H}-\mathrm{SiC}(0001)$, one goal was to find a way to efficiently decouple the graphene from its substrate. Hydrogen intercalation below graphene/SiC(0001) was first demonstrated by C. Riedl et al. [149]. They showed that annealing graphene/ $\mathrm{SiC}(0001)$ at $900 \mathrm{~K}$ in presence of $H_{2}$ gas at atmospheric pressure resulted in intercalation of Hydrogen atoms down to the $\mathrm{SiC}$ interface, leading to decouple the buffer layer from its $\mathrm{SiC}$ substrate. Moreover, Hydrogen atoms passivated $\mathrm{Si}$ atoms of $\mathrm{SiC}(0001)$ substrate and they obtained quasi-freestanding graphene layer undoped with $E_{f}=E_{D}$. By exposing buffer layer $/ \mathrm{SiC}(0001)$ to $\mathrm{O}_{2}$ gas at atmospheric pressure while annealing at 520K, S. Oida et al. [150] have shown that intercalation of Oxygen molecules led to the formation of a thin $(\sim 3 \AA)$ amorphous oxide layer at buffer layer/SiC interface thus decoupling the buffer layer from the substrate.

Moreover, chemical reactions may take place at the interlayer space between graphene and its substrate : oxidation of intercalated CO molecules between graphene and $\mathrm{Pt}(111)$ [151], chemical reaction between intercalated $\mathrm{O}$ et $\mathrm{H}_{2}$ atoms below graphene/Pt(111) [91], or formation of a 2D GaN layer between monolayer graphene and $\mathrm{SiC}(0001)$ [152]. In this case, they first intercalated Hydrogen to obtain quasi-freestanding bilayer graphene/ $\mathrm{SiC}(0001)$ which provides a confined and passivated space that may promote Frank-van der Merve growth of GaN. Using MOCVD and annealing at 820K, Ga atoms were intercalated at the $\mathrm{SiC}$ interface. Decomposition at $950 \mathrm{~K}$ of Ammonia induced chemical reaction under graphene layers between intercalated Ga and atomic $\mathrm{N}$ atoms, leading to the formation of $2 \mathrm{D}$ Ga $N_{x}$ layer covalently bound to $\mathrm{SiC}(0001)$.

\section{Transition metal dichalcogenides materials and intercalation}

Transition metal dichalcogenides (TMDs : $\mathrm{M} X_{2}$ where $\mathrm{M}=\mathrm{Mo}, \mathrm{W} ; \mathrm{X}=$ $\mathrm{S}, \mathrm{Se}, \mathrm{Te}$ ) are layered semiconducting materials which have attracted considerable attention because their electronic properties are significantly influenced by their single-layer limit $[154,155]$. Unlike graphene, TMDs have a large bandgap which makes these materials more versatile candidates for device applications. More interestingly, these materials show a transition from an indirect bandgap to a larger direct bandgap when going from bulk to monolayer [156]. Because of this direct bandgap, 2D TMDs present strong photoluminescence properties. They are then more suitable for optoelectronic applications [157] and field-effect transistors with high on/off ratio compared 


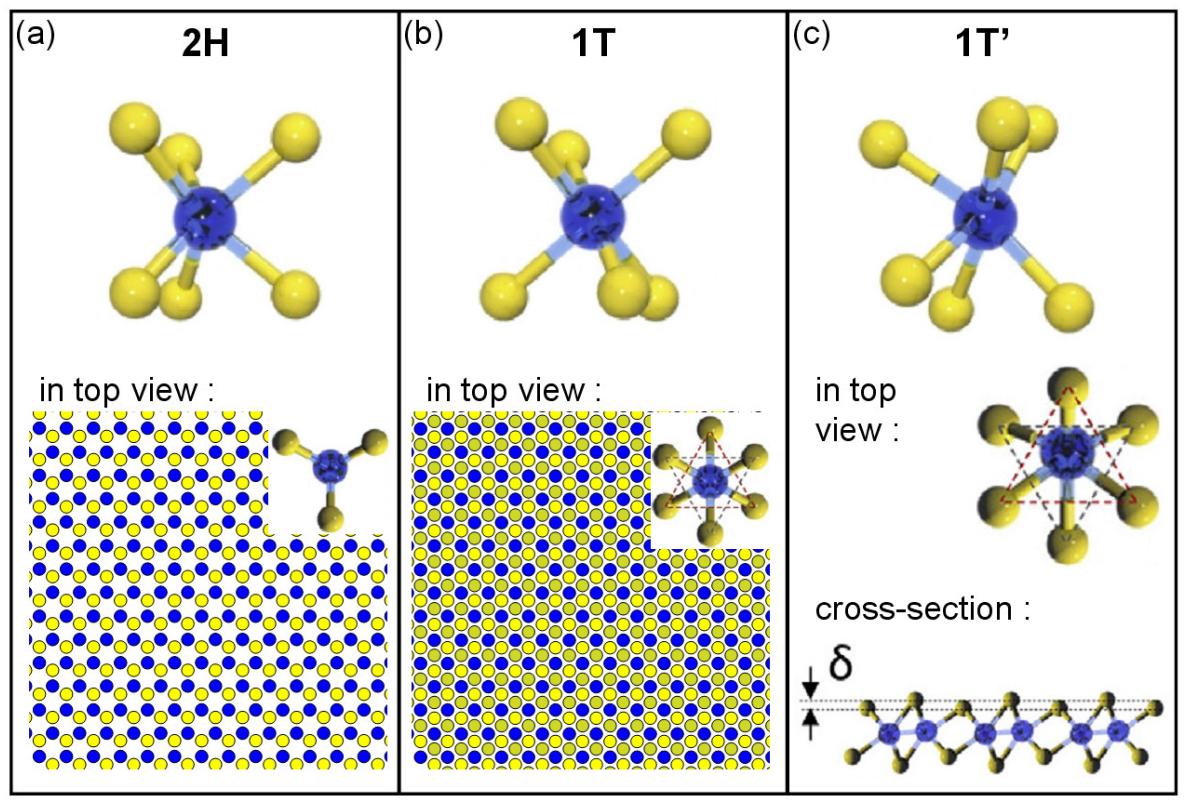

Figure 9: This figure presents three polymorphic forms of 2D TMDs, namely $2 \mathrm{H}, 1 \mathrm{~T}$ and 1T'. (a) In $2 \mathrm{H}$, each $\mathrm{M}$ atom (in blue) is coordinated by six $\mathrm{X}$ atoms (in yellow) in a trigonal prismatic geometry. (b) $1 \mathrm{~T}$-form shows the two prims of the $2 \mathrm{H}$-form coordinating $\mathrm{M}$ and $\mathrm{X}$ atoms, one being rotated by $180^{\circ}$ with respect to the other one. To clearly see it in the top view, the two chalcogen layers are drawn in slightly different colors. (c) $1 T^{\prime}$-form is a distorted form of $1 \mathrm{~T}$-form, with atomic displacement of $\mathrm{X}$ atoms along the vertical direction, as seen in the cross-section view. This figure is adapted from Ref. [153] with permission. 
to graphene-based devices [158, 159]. For example, 2D Molybdenum disulfide $\left(\mathrm{Mo} S_{2}\right)$ presents a direct gap of $1.8 \mathrm{eV}$ (decreasing to an indirect bandgap of around $1.2 \mathrm{eV}$ in bulk) [160,161], a good mobility (at least $200 \mathrm{~cm}^{2} V^{-1} \mathrm{~s}^{-1}$ at room temperature) and a high current on/off ratio of around $10^{8}$ [159]. Moreover, 2D TMDs are promising candidates for a large range of applications, from sensors, to energy storage or piezoelectric devices [153]. Finally, it has been predicted theoretically the existence of SHE and non-trivial SHE effects in 2D TMDs due to strong SO coupling and the breaking of inversion symmetry in their single layer form, raising high expectations for spin-physics exploration $[162,163,164]$.

2D TMDs are composed of three layers strongly bound, where the transition metal $(\mathrm{M})$ hexagonal $1 \times 1$ monolayer is sandwiched between two chalcogen (X) hexagonal $1 \times 1$ monolayers. Each $\mathrm{M}$ atom is coordinated to six neighboring $\mathrm{X}$ atoms. TMDs possess different polymorphic forms, such as $2 \mathrm{H}, 1 \mathrm{~T}$ and 1T', schematized in Fig. 9, where 2H-form is usually the most stable phase at room temperature. Electronic properties of 2D TMDs vary greatly with their polymorphic forms : for example, in the case of $\mathrm{Mo} S_{2}, 2 \mathrm{H}$-form is semiconducting whereas the $1 \mathrm{~T}$-form is metallic $[165,166]$. In a very recent work, B. Pal et al. [167] have shown a metastable semiconducting 1T'-form of $\mathrm{Mo} S_{2}$ with a small gap of $90 \pm 40 \mathrm{meV}$. This is in contrast to most assertions in the scientific literature suggesting that the metastable phase is metallic.

Various top-down and bottom-up methods have been used in order to synthesize large-scale 2D TMDs layer of high quality (defectivless and uniform), controlling precisely the number of layers and on various substrates. This still remains very challenging and thus new methods are always tested. As in bulk TMDs interactions between each X-M-X layer are the weak van der Waals ones, exfoliation methods such as mechanical or liquid-based exfoliation, were first used. The state-of-art mechanical exfoliation method produces high quality 2D TMDs, but their sizes and their uniformity is not satisfactory for mass production or devices. Bottom-up methods for producing high quality, wafer-scale 2D TMDs include Chemical Vapor Deposition (CVD) method [168, 169, 170], Metal-Organic-CVD (MOCVD) method, Atomic Layer Deposition (ALD) method and direct deposition methods, such as sputtering $[171,172,173,153]$. Another quite "exotic" method has been reported by T. Eknapakul et al. [164] who have obtained a quasi-freestanding Mo $S_{2}$ monolayer on its bulk crystal using Potassium intercalation. Indeed, after deposition of $\mathrm{K}$ atoms on top of the sample without annealing, the band structure measured by ARPES was modified due to intercalation and revea- 
led a transition from bulk $\operatorname{Mo} S_{2}$ to an isolated monolayer of $\operatorname{Mo} S_{2}$. Contrary to the mechanical exfoliation method, liquid-based exfoliation strategy presents great advantages for scalability and mass production [174]. Intercalation chemistry plays a major role in this method, as it must promote the insertion of foreign species between individual 2D layers thus increasing the interlayer space, without damaging the TMDs layers (see Ref. [175] for a review about liquid-based exfoliation method). In addition to exfoliate individual layers, intercalation may also modify the atomic, electronic and structural properties of host material and lead to other remarkable properties, such as drastic changes in conductivity or superconductivity [154]. For example, upon Lithium intercalation $2 \mathrm{H}$-form $\mathrm{MoS}_{2}$ undergoes a first order phase transition to $1 \mathrm{~T}$ - and $1 \mathrm{~T}$ '-forms $[176,165,177,166,178,179]$. This induces an increase of charge carrier conductivity and improves the catalytic reactivity of the surface. The coexistence of semiconducting $2 \mathrm{H}$ - with metallic 1T- and 1T'-phases results in unusual transport phenomena [179]. Intercalating TMDs with ions may lead to superconductivity. Indeed, R.B. Somoano et al. [180] have shown that intercalating alkali metal atoms (Li, Na, K, $\mathrm{Rb}$ and $\mathrm{Cs}$ ) into single crystals of $\mathrm{MoS}_{2}$ resulted in superconductivity. The superconductivity is believed to be largely due to the charge transfer from alkali metal atoms to $\mathrm{Mo} S_{2}$, resulting in an increase in electron density and density of states at the Fermi surface.

\section{Conclusion}

The possibility to intercalate molecules (even large) and atoms between 2D layers of a stacked van der Waals heterostructure opens huge research fields for the realization of new materials with novel properties. Among the $2 \mathrm{D}$ systems, the pioneering graphene, is the most studied and a lot of fascinating physics start to be discovered : new types of superconductivity, the possibility to realize topological phase by introducing spin-orbit coupling and playing with new types of Dirac fermions. Concerning the other 2D materials particularly TMDs, their functionalization by intercalation is still realized in liquid phase and, like at the beginning of graphene, chemical methods are used to exfoliate and prepare monolayer of isolated TMDs. The studies of modifications of their physical properties, e.g. by following changes in the band structure, remain largely unexplored. 


\section{References}

[1] A.H. Castro Neto, F. Guinea, N.M.R. Peres, K.S. Novoselov, A.K. Geim, The electronic properties of graphene, Rev. Mod. Phys. 81 (2009) 109-162, and references therein.

[2] K.S. Novoselov, A.K. Geim, S.V. Morozov, D. Jiang, Y. Zhang, S.V. Dubonos, I.V. Grigorieva, A.A. Firsov, Electric Field Effect in Atomically Thin Carbon Films, Science 306 (2004) 666-669.

[3] B. Dlubak, M.-B. Martin, C. Deranlot, B. Servet, S. Xavier, R. Mattana, M. Sprinkle, C. Berger, W.A. De Heer, F. Petroff, A. Anane, P. Seneor, A. Fert, Highly efficient spin transport in epitaxial graphene on SiC, Nat. Phys. 8 (2012) 557-561.

[4] K.S. Novoselov, Z. Jiang, Y. Zhang, S.V. Morozov, H.L. Stormer, U. Zeitler, J.C. Maan, G.S. Boebinger, P. Kim, A.K. Geim, Roomtemperature quantum Hall effect in graphene, Science 315 (2007) 1379.

[5] K.S. Novoselov, A.K. Geim, S.V. Morozov, D. Jiang, M.I. Katsnelson, I.V. Grigorieva, S.V. Dubonos, A.A. Firsov, Two-dimensional gas of massless Dirac fermions in graphene, Nature 438 (2005) 197-200.

[6] J.-S. Park, H.J. Choi, Band-gap opening in graphene : A reverseengineering approach, Phys. Rev. B 92 (2015) 045402.

[7] S. Manzeli, D. Ovchinnikov, D. Pasquier, O.V. Yazyev, A. Kis, 2D transition metal dichalcogenides, Nat. Rev. Mat. 2 (2017) 17033.

[8] P. Vogt, P. De Padova, C. Quaresima, J. Avila, E. Frantzeskakis, M.C. Asensio, A. Resta, B. Ealet, G. Le Lay, Silicene : Compelling Experimental Evidence for Graphenelike Two-Dimensional Silicon, Phys. Rev. Lett. 108 (2012) 155501.

[9] H. Oughaddou, H. Enriquez, M. R. Tchalala, H. Yildirim, A. J. Mayne, A. Bendounan, G. Dujardin, M. Ait Ali and A. Kara, Silicene, a promising new 2D material. Progress in Surface Science90 (2015), 46-83.

[10] M. Derivaz, D. Dentel, R. Stephan, M.-C. Hanf, A. Mehdaoui, P. Sonnet, C. Pirri, Continuous Germanene Layer on Al(111), NanoLett. 15 (2015) 2510-2516. 
[11] F.-F. Zhu, W.-J. Chen, Y. Xu, C.-L. Gao, D.-D. Guan, C.-H. Liu, D. Qian, S.-C. Zhang, J.-F. Jia, Epitaxial growth of two-dimensional stanene, Nat. Mat. 14 (2015) 1020-1025.

[12] A. Favron, E. Gaufrès, F. Fossard, A.-L. Phaneuf-L'Heureux, N.Y.-W. Tang, P.L. Lévesque, A. Loiseau, R. Leonelli, S. Francoeur, R. Martel, Photooxidation and quantum confinement effects in exfoliated black phosphorus, Nat. Mat. 14 (2015) 826-832.

[13] A.J. Mannix, X.-F. Zhou, B. Kiraly, J.D. Wood, D. Alducin, B.D. Myers, X. Liu, B.L. Fisher, U. Santiago, J.R. Guest, M.J. Yacaman, A. Ponce, A.R. Oganov, M.C. Hersam, N.P. Guisinger, Synthesis of borophenes : Anisotropic, two-dimensional boron polymorphs, Science 350 (2015) 1513-1516.

[14] S.K. Mahatha, P. Moras, P.M. Sheverdyaeva, V. Bellini, T.O. Menteş, A. Locatelli, R. Flammini, K. Horn, C. Carbone, Absence of Dirac cones in monolayer silicene and multilayer Si films on $\mathrm{Ag}(111)$, J. Elec. Spec. Rel. Phenom. 219 (2017) 2-8.

[15] S. Sadeddine, H. Enriquez, A. Bendounan, PK. Das, I. Vobornik, A. Kara, A.J. Mayne, F. Sirroti, G. Dujardin and H. Oughaddou, Compelling experimental evidence of a Dirac cone in the electronic structure of a 2D silicon layer, Scientific Reports 7 (2017) 44400.

[16] C. Bena, L. Simon, Dirac point metamorphosis from third-neighbor couplings in graphene and related materials, Phys Rev. B 83 (2011) 115404.

[17] C.-H. Park, L. Yang, Y.-W. Son, M.L. Cohen, S.G. Louie, Anisotropic behaviors of massless Dirac fermions in graphene under periodic potentials, Nat. Phys. 4 (2008) 213-217.

[18] J.G. Pedersen, A.W. Cummings, S. Roche, Anisotropic behavior of quantum transport in graphene superlattice : Coexistence of ballistic conduction with Anderson insulating regime, Phys. Rev. B 89 (2014) 165401.

[19] G. Trambly de Laissardière, D. Mayou, L. Magaud, Localization of Dirac Electrons in Rotated Graphene Bilayers, Nano Lett. 10 (2010) 804-810. 
[20] R. Tsu, Superlattice to Nanoelectronics, Elsevier, Oxford, UK 2005.

[21] M.G. Cottam, D.R. Tilley, Introduction to Surface and Superlattice Excitations, Cambridge Uni. Press, Cambridge, UK 1989.

[22] A. Sinitskii, J.M. Tour, Patterning Graphene through the SelfAssembled Templates: Toward Periodic Two-dimensional Graphene Nanostructures with Semiconductor Properties, J. Am. Chem. Soc. 132 (2010) 14730-14732.

[23] C. Coletti, C. Riedl, D.S. Lee, B. Krauss, L. Patthey, K. von Klitzing, J.H. Smet, U. Starke, Charge neutrality and band-gap tuning of epitaxial graphene on $\mathrm{SiC}$ by molecular doping, Phys. Rev. B 81 (2010) 235401.

[24] R. Philipson, C.J. Lockhart de la Rosa, J. Teyssandier, P. Walke, D. Waghray, Y. Fujita, J. Adisoejoso, K.S. Mali, I. Asselberghs, C. Huyghebaert, H. Uji-i, S. De Gendt, S. De Feyter, Tunable doping of graphene by using physisorbed self-assembled networks, Nanoscale 8 (2016) 20017-20026.

[25] M. Gobbi, S. Bonacchi, J.X. Lian, Y. Liu, X.-Y. Wang, M.-A. Stoeckel, M.A. Squillaci, G. D'Avino, A. Narita, K. Müllen, X. Feng, Y. Olivier, D. Beljonne, P. Samorì, E. Orgiu, Periodic potentials in hybrid van der Waals heterostructures formed by supramolecular lattices on graphene, Nat. Commun. 8 (2017) 14767.

[26] L. Daukiya, C. Mattioli, D. Aubel, S. Hajjar-Garreau, F. Vonau, E. Denys, G. Reiter, J. Fransson, E. Perrin, M.-L. Bocquet, C. Bena, A. Gourdon, L. Simon, Covalent Functionalization by Cycloaddition Reactions of Pristine Defect-Free Graphene, ACS Nano 11 (2017) 627-634.

[27] M. Dubois, K. Guérin, Y. Ahmad, N. Batisse, M. Mar, L. Frezet, W. Hourani, J.-L. Bubendorff, J. Parmentier, S. Hajjar-Garreau, L. Simon, Thermal exfoliation of fluorinated graphite, Carbon 77 (2014) 688-704.

[28] H. Li, L. Daukiya, S. Haldar, A. Lindblad, B. Sanyal, O. Eriksson, D. Aubel, S. Hajjar-Garreau, L. Simon, K. Leifer, Site-selective local fluorination of graphene induced by focused ion beam irradiation, Nat. Scient. Rep. 6 (2016) 19719. 
[29] C. Riedl, A.A. Zakharov, U. Starke, Precise in situ thickness analysis of epitaxial graphene layers on $\mathrm{SiC}(0001)$ using low-energy electron diffraction and angle resolved ultraviolet photoelectron spectroscopy, Appl. Phys. Lett. 93 (2008) 033106.

[30] K.V. Emtsev, A.A. Zakharov, C. Coletti, S. Forti, U. Starke, Ambipolar doping in quasifree epitaxial graphene on $\mathrm{SiC}(0001)$ controlled by Ge intercalation, Phys. Rev. B 84 (2011) 125423.

[31] I. Gierz, T. Suzuki, R.T. Weitz, D.S. Lee, B. Krauss, C. Riedl, U. Starke, H. Höchst, J.H. Smet, C.R. Ast, K. Kern, Electronic decoupling of an epitaxial graphene monolayer by gold intercalation, Phys. Rev. B 81 (2010) 235408.

[32] A. Varykhalov, J. Sánchez-Barriga, A.M. Shikin, C. Biswas, E. Vescovo, A. Rybkin, D. Marchenko, O. Rader, Electronic and Magnetic Properties of Quasifreestanding Graphene on Ni, Phys. Rev. Lett. 101 (2008) 157601.

[33] A. Varykhalov, M.R. Scholz, T.K. Kim, O. Rader, Effect of noble-metal contacts on doping and band gap of graphene, Phys. Rev. B 82 (2010) 121101(R).

[34] P. Sutter, P. Albrecht, X. Tong, E. Sutter, Mechanical Decoupling of Graphene from $\mathrm{Ru}(0001)$ by Interfacial Reaction with Oxygen, J. Phys. Chem. C 117 (2013) 6320-6324.

[35] P. Lauffer, K.V. Emtsev, R. Graupner, Th. Seyller, L. Ley, S.A. Reshanov, H.B. Weber, Atomic and electronic structure of few-layer graphene on $\mathrm{SiC}(0001)$ studied with scanning tunneling microscopy and spectroscopy, Phys. Rev. B 77 (2008) 155426.

[36] L. Daukiya, M.N. Nair, S. Hajjar-Garreau, F. Vonau, D. Aubel, J.L. Bubendorff, M. Cranney, E. Denys, A. Florentin, G. Reiter, L. Simon, Highly n-doped graphene generated through intercalated terbium atoms, Phys. Rev. B 97 (2018) 035309.

[37] L. Simon, J.-L. Bischoff, L. Kubler, X-ray photoelectron characterization of 6H-SiC(0001), Phys. Rev. B 60 (1999) 11653. 
[38] P. Mallet, F. Varchon, C. Naud, L. Magaud, C. Berger, J.-Y. Veuillen, Electron states of mono- and bilayer graphene on $\mathrm{SiC}$ probed by scanning-tunneling microscopy, Phys. Rev. B 76 (2007) 041403(R).

[39] C. Riedl, C. Coletti, U. Starke, Structural and electronic properties of epitaxial graphene on $\mathrm{SiC}(0001)$ : a review of growth, characterization, transfer doping and hydrogen intercalation, J. Phys. D : Appl. Phys. 43 (2010) 374009.

[40] M.S. Nevius, M. Conrad, F. Wang, A. Celis, M.N. Nair, A. TalebIbrahim, A. Tejeda, E.H. Conrad, Semiconducting Graphene from highly ordered substrate interaction, Phys. Rev. Lett. 115 (2015) 136802 .

[41] R. Balog, B. Jørgensen, L. Nilsson, Mie Andersen, E. Rienks, M. Bianchi, M. Fanetti, E. Lægsgaard, A. Baraldi, S. Lizzit, Z. Slijvancanin, F. Besenbacher, B. Hammer, T.G. Pedersen, P. Hofmann, L. Hornkær, Bandgap opening in graphene induced by patterned hydrogen adsorption, Nat. Mat. 9 (2010) 315-319.

[42] R. Grassi, T. Low, M. Lundstrom, Scaling of the energy gap in patternhydrogenated graphene, Nano Lett. 11 (2011) 4574-4578.

[43] N.R. Gall, E.V. Rut'kov, A. Ya. Tontegode, Two Dimensional Graphite Films on Metals and their Intercalation, Int. J. Mod. Phys. 11 (1997) 1865-1911.

[44] L. Huang, Y. Pan, L. Pan, M. Gao, W. Xu, Y. Que, H. Zhou, Y. Wang, S. Du, H.-J. Gao, Intercalation of metal islands and films at the interface of epitaxially grown graphene and $\mathrm{Ru}(0001)$ surfaces, Appl. Phys. Lett. 99 (2011) 163107.

[45] T. Gao, Y. Gao, C. Chang, Y. Chen, M. Liu, S. Xie, K. He, X. Ma, Y. Zhang, Z. Liu, Atomic-Scale Morphology and electronic structure of manganese atomic layer underneath epitaxial graphene on $\mathrm{SiC}(0001)$, ACS Nano 6 (2012) 6562-6568.

[46] S.J. Sung, J.W. Yang, P.R. Lee, J.G. Kim, M.T. Ryu, H.M. Park, G. Lee, C.C. Hwang, Kwang.S. Kim, J.S. Kim, J.W. Chung, Spin-induced band modifications of graphene through intercalation of magnetic iron atoms, Nanoscale 6 (2014) 3824-3829. 
[47] Y. Zhang, H. Zhang, Y. Cai, J. Song, P. He, The investigation of cobalt intercalation underneath epitaxial graphene on $6 \mathrm{H}-\mathrm{SiC}(0001)$, Nanotech. 28 (2017) 075701.

[48] K. Yagyu, T. Tajiri, A. Kohno, K. Takahashi, H. Tochihara, H. Tomokage, T. Suzuki, Fabrication of a single layer graphene by copper intercalation on a $\mathrm{SiC}(0001)$ surface, Appl. Phys. Lett. 104 (2014) 053115.

[49] C. Xia, A.A. Tal, L.I. Johansson, W. Olovsson, I.A. Abrikosov, C. Jacobi, Effects of rhenium on graphene grown on $\mathrm{SiC}(0001)$, J. Elec. Spectr. Rel. Phen. 222 (2017) 117-121.

[50] B. Premlal, M. Cranney, F. Vonau, D. Aubel, D. Casterman, M.M. De Souza, L. Simon, Surface intercalation of gold underneath a graphene monolayer on $\mathrm{SiC}(0001)$ studied by scanning tunneling microscopy and spectroscopy, Appl. Phys. Lett. 94 (2009) 263115.

[51] I. Gierz, C. Riedl, U. Starke, C.R. Ast, K. Kern, Atomic Hole Doping of Graphene, Nano Lett. 8 (2008) 4603-4607.

[52] A.G. Starodubov, M.A. Medvetskii, A.M. Shikin, V.K. Adamchuk, Intercalation of Silver Atoms under a Graphite Monolayer on $\mathrm{Ni}(111)$, Phys. Sol. State 46 (2004) 1340-1348.

[53] M. Papagno, P. Moras, P.M. Sheverdyaeva, J. Doppler, A. Garhofer, F. Mittendorfer, J. Redinger, C. Carbone, Hybridization of graphene and a Ag monolayer supported on $\operatorname{Re}(0001)$, Phys. Rev. B 88 (2013) 235430 .

[54] C. Xia, L.I. Johansson, A.A. Zakharov, L. Hultman, C. Virojanadara, Effects of $\mathrm{Al}$ on epitaxial graphene grown on $6 \mathrm{H}-\mathrm{SiC}(0001)$, Mat. Res. Exp. 1 (2014) 015606.

[55] Y.R. Niu, A.A. Zakharov, R. Yakimova, Metal-dielectric transition in Sn-intercalated graphene on $\mathrm{SiC}(0001)$, Ultramicro. 183 (2017) 49-54.

[56] S. Hayashi, A. Visikovskiy, T. Kajiwara, T. Iimori, T. Shirasawa, K. Nakastuji, T. Miyamachi, S. Nakashima, K. Yaji, K. Mase, F. Komori, S. Tanaka, Triangular lattice atomic layer of $\mathrm{Sn}(1 \mathrm{x} 1)$ at graphene/SiC(0001) interface, Appl. Phys. Expr. 11 (2018) 015202. 
[57] A. Yurtsever, J. Onoda, T. Iimori, K. Niki, T. Miyamachi, M. Abe, S. Mizuno, S. Tanaka, F. Komori, Y. Sugimoto, Effects of Pb Intercalation on the Structural and Electronic Properties of Epitaxial Graphene on SiC, Small 12 (2016) 3955-3966.

[58] C. Xia, S. Watcharinyanon, A.A. Zakharov, R. Yakimova, L. Hultman, L.I. Johansson, C. Virojanadara, Si intercalation/deintercalation of graphene on 6H-SiC(0001), ACSNano 6 (2012) 6562-6568.

[59] I.I. Klimovskikh, M.M. Otrokov, V.Yu. Voroshnin, D. Sostina, L. Petaccia, G. Di Santo, S. Thakur, E.V. Chulkov, A.M. Shikin, Spin-Orbit Coupling Induced Gap in Graphene on Pt(111) with Intercalated Pb Monolayer, ACS Nano 11 (2017) 368-374.

[60] D.A. Estyunin, I.I. Klimovskikh, V.Yu. Voroshnin, D.M. Sostina, L. Petaccia, G. Di Santo, A.M. Shikin, Formation of a quasi-free-standing graphene with a band gap at the dirac point by $\mathrm{Pb}$ atoms intercalation under graphene on Re(0001), J. Exp. Theo. Phys. 125 (2017) 762-767.

[61] A.L. Walter, K.-J. Jeon, A. Bostwick, F. Speck, M. Ostler, T. Seyller, L. Moreschini, Y.S. Kim, Y.J. Chang, K. Horn, E. Rotenberg, Highly p-doped epitaxial graphene obtained by fluorine intercalation, Appl. Phys. Lett. 98 (2011) 184102.

[62] M.S. Dresselhaus, G. Dresselhaus, Intercalation compounds of graphite, Adv. Phys. 51 (2002) 1-186.

[63] D. Appy, H. Lei, C-Z. Wang, M. C. Tringides, D.-J Liu, J. W. Evans, P. A. Thiel, A. Transition metals on the (0001) surface of graphite : Fundamental aspects of adsorption, diffusion, and morphology. Progress in Surface Science 89(2014) 219-238.

[64] T. Minato, T. Abe, Surface and interface sciences of Li-ion batteries. Progress in Surface Science 92 2017, 240-280.

[65] T.E. Weller, M. Ellerby, S.S. Saxena, R.P. Smith, N.T. Skipper, Superconductivity in the intercalated graphite compounds $C_{6} \mathrm{Yb}$ and $C_{6} \mathrm{Ca}$, Nat. Phys. 1 (2005) 39-41. 
[66] N. Emery, C. Hérold, M. d'Astuto, V. Garcia, Ch. Bellin, J.F. Marêché, P. Lagrange, G. Loupias, Superconductivity of Bulk $\mathrm{Ca}_{6}$, Phys. Rev. Lett. 95 (2005) 087003.

[67] S. Heguri, N. Kawade, T. Fujisawa, A. Yamaguchi, A. Sumiyama, K. Tanigaki, M. Kobayashi, Superconductivity in the Graphite Intercalation Compound $\mathrm{BaC}_{6}$, Phys. Rev. Lett. 114 (2015) 247201.

[68] A.Ya. Tontegode, Carbon on Transition Metal Surfaces, Prog. Surf. Sci. 38 (1991) 201-429.

[69] K. Sugawara, K. Kanetani, T. Sato, T. Takahashi, Fabrication of Liintercalated bilayer graphene, AIP Adv. 1 (2011) 022103.

[70] C. Virojanadara, S. Watcharinyanon, A.A. Zakharov, L.I. Johansson, Epitaxial graphene on $6 \mathrm{H}-\mathrm{SiC}$ and $\mathrm{Li}$ intercalation, Phys. Rev. B 82 (2010) 205402.

[71] S. Ichinokura, K. Sugawara, A. Takayama, T. Takahashi, S. Hasegawa, Superconducting Calcium-Intercalated Bilayer Graphene, ACS Nano 10 (2016) 2761-2765.

[72] S. Watcharinyanon, , C. Virojanadara, L.I. Johansson, Rb and Cs deposition on epitaxial graphene grown on $6 \mathrm{H}-\mathrm{SiC}(0001)$, Surf. Sci. 605 (2011) 1918-1922.

[73] M. Petrović, I. Šrut Rakić, S. Runte, C. Busse, J.T. Sadowski, P. Lazić, I. Pletikosić, Z.-H. Pan, M. Milun, P. Pervan, N. Atodiresei, R. Brako, D. Šokčević, T. Valla, T. Michely, M. Kralj, The mechanism of caesium intercalation of graphene, Nat. Comm. 4 (2013) 2772.

[74] M. Calandra, F. Mauri, Theoretical explanation of superconductivity in $C_{6}$ Ca, Phys. Rev. Lett. 95 (2005) 237002.

[75] R.P. Smith, Th.E. Weller, C.A. Howard, M.P.M. Dean, K.C. Rahnejat, S.S. Saxena, M. Ellerby, Superconductivity in graphite intercalation compounds, Phys. C 514 (2015) 50-58.

[76] S. Sung, S. Kim, P. Lee, J. Kim, M. Ryu, H. Park, K. Kim, B.I. Min, J. Chung, Observation of variable hybridized-band gaps in Euintercalated graphene, Nanotech. 28 (2017) 205201. 
[77] S. Watcharinyanon, L.I. Johansson, C. Xia, J. Ingo Flege, A. Meyer, J. Falta, C. Virojanadara, Ytterbium Intercalation of Epitaxial Graphene Grown on Si-Face SiC, Graphene 2 (2013) 66-73.

[78] J. Kim, P. Lee, M. Ryu, H. Park, J. Chung, Cerium-induced changes in the $\pi$-band of graphene, RSC Adv. 6 (2016) 114219-114223.

[79] S. Schumacher, T.O. Wehling, P. Lazić, S. Runte, D.F. Förster, C. Busse, M. Petrović, M. Kralj, S. Blüügel, N. Atodiresei, V. Caciuc, T. Michely, The Backside of Graphene : Manipulating Adsorption by Intercalation, Nano Lett. 13 (2013) 5013-5019.

[80] B. Hunt, J.D. Sanchez-Yamagishi, A.F. Young, M. Yankowitz, B.J. LeRoy, K. Watanabe, T. Taniguchi, P. Moon, M. Koshino, P. JarilloHerrero, R.C. Ashoori, Massive Dirac Fermions and Hofstadter Butterfly in a van der Waals Heterostructure, Science 340 (2013) 1427-1430.

[81] A.M. Shikin, G.V. Prudnikova, V.K. Adamchuk, F. Moresco, K.-H. Rieder, Surface intercalation of gold underneath a graphite monolayer on $\mathrm{Ni}(111)$ studied by angle-resolved photoemission and high-resolution electron-energy-loss spectroscopy, Phys. Rev. B 62 (2000) 13202.

[82] M. Cranney, F. Vonau, P.B. Pillai, E. Denys, D. Aubel, M.M. De Souza, C. Bena, L. Simon, Superlattice of resonators on monolayer graphene created by intercalated gold nanoclusters, EuroPhys. Lett. 91 (2010) 66004 .

[83] L. Simon, C. Bena, F. Vonau, M. Cranney, D. Aubel, Fourier-transform scanning tunnelling spectroscopy : the possibility to obtain constantenergy maps and band dispersion using a local measurement, J. Phys. D : Appl. Phys. 44 (2011) 464010.

[84] M.N. Nair, M. Cranney, F. Vonau, D. Aubel, P. Le Fèvre, A. Tejeda, F. Bertran, A. Taleb-Ibrahimi, L. Simon, High Van Hove singularity extension and Fermi velocity increase in epitaxial graphene functionalized by intercalated gold clusters, Phys. Rev. B 85 (2012) 245421.

[85] M. Narayanan Nair, M. Cranney, T. Jiang, S. Hajjar-Garreau, D. Aubel, F. Vonau, A. Florentin, E. Denys, M.-L. Bocquet, L. Simon, Noblemetal intercalation process leading to a protected adatom in a graphene hollow site, Phys. Rev. B 94 (2016) 075427. 
[86] I. Horcas, R. Fernández, J.M. Gómez-Rodríguez, J. Colchero, J. Gómez-Herrero, A.M. Baro, WSXM : A software for scanning probe microscopy and a tool for nanotechnology, Rev. Sci. Instrum. 78 (2007) 013705.

[87] M. Vanin, J.J. Mortensen, A.K. Kelkkanen, J.-M. Garcia-Lastra, K.S. Thygesen, K.W. Jacobsen, Graphene on metals : A van der Waals density functional study, Phys. Rev. B 81 (2010) 081408.

[88] M. Neek-Amal, R. Asgari, M.R. Rahimi Tabar, The formation of atomic nanoclusters on graphene sheets, Nanotech. 20 (2009) 135602.

[89] X. Liu, Y. Han, J.W. Evans, A.K. Engstfeld, R.J. Behm, M.C. Tringides, M. Hupalo, H.-Q. Lin, L. Huang, K.-M. Ho, D. Appy, P.A. Thiel, C.-Z. Wang, Growth morphology and properties of metals on graphene, Prog. Surf. Sci. 90 (2015) 397-443.

[90] J.B. Hannon, R.M. Tromp, Pit formation during graphene synthesis on $\mathrm{SiC}(0001)$ : In situ electron microscopy, Phys. Rev. B 77 (2008) 241404 .

[91] Q. Fu, X. Bao, Surface chemistry and catalysis confined under twodimensional materials, Chem. Soc. Rev. 46 (2017) 1842-1874.

[92] N.R. Gall, E.V. Rut'kov, A.Ya. Tontegode, M.M. Usufov, Regularities of intercalation of two-dimensional graphite films on metals by atoms and $C_{60}$ molecules, Synth. Met. 97 (1998) 171-173.

[93] J. Hass, W.A. de Heer, E.H. Conrad, The growth and morphology of epitaxial multilayer graphene, J. Phys. Cond. Matt. 20 (2008) 323202.

[94] N. Ferralis, R. Maboudian, C. Carraro, Evidence of Structural Strain in Epitaxial Graphene Layers on 6H-SiC(0001), Phys. Rev. Lett. 101 (2008) 156801.

[95] A. Udupa, A. Martini, Model predictions of shear strain-induced ridge defects in graphene, Carbon 49 (2011) 3571-3578.

[96] C. Hérold, P. Lagrange, Composés d'intercalation du graphite : des binaires aux ternaires, C. R. Chimie 6 (2003) 457-465. 
[97] F. Vonau, D. Aubel, G. Gewinner, C. Pirri, J.C. Peruchetti, D. Bolmont, L. Simon, Fermi contour imaging of the two-dimensional semimetal Er $S i_{2}$ by Fourier transform STM, Phys. Rev. B 69 (2004) 081305.

[98] J.L. McChesney, A. Bostwick, T. Ohta, T. Seyller, K. Horn, J. González, E. Rotenberg, Extended Van Hove Singularity and Superconducting Instability in Doped Graphene, Phys. Rev. Lett. 104 (2010) 136803.

[99] A. Bostwick, F. Speck, T. Seyller, K. Horn, M. Polini, R. Asgari, A.H. MacDonald, E. Rotenberg, Observation of Plasmarons in QuasiFreestanding Doped Graphene, Science 328 (2010) 999-1002.

[100] D. Bohm, D. Pines, A Collective Description of Electron Interactions : III. Coulomb Interactions in a Degenerate Electron Gas, Phys. Rev. 92 (1953) 609.

[101] V.N. Kotov, B. Uchoa, V.M. Pereira, F. Guinea, A.H. Castro Neto, Electron-Electron Interactions in Graphene : Current Status and Perspectives, Rev. Mod. Phys. 84 (2012) 1067.

[102] A.M. Shikin, A.G. Rybkin, D. Marchenko, A.A. Rybkina, M.R. Scholz, O. Rader, A. Varykhalov, Induced spin-orbit splitting in graphene : the role of atomic number of the intercalated metal and $\pi-\mathrm{d}$ hybridization, New J. Phys. 15 (2013) 013016.

[103] D. Marchenko, A. Varykhalov, M.R. Scholz, G. Bihlmayer, E.I. Rashba, A. Rybkin, A.M. Shikin, O. Rader, Giant Rashba splitting in graphene due to hybridization with gold, Nat. Comm. 3 (2012) 1232.

[104] D. Marchenko, A. Varykhalov, J. Sánchez-Barriga, Th. Seyller, O. Rader, Rashba splitting of $100 \mathrm{meV}$ in Au-intercalated graphene on $\mathrm{SiC}$, Appl. Phys. Lett. 108 (2016) 172405.

[105] C.L. Kane, E.J. Mele, Quantum Spin Hall Effect in Graphene, Phys. Rev. Lett. 95 (2005) 226801.

[106] C. Weeks, J. Hu, J. Alicea, M. Franz, R. Wu, Engineering a Robust Quantum Spin Hall State in Graphene via Adatom Deposition, Phys. Rev X 1 (2011) 021001. 
[107] Y. Wang, X. Cai, J. Reutt-Robey, M.S. Fuhrer, Neutral-current Hall effects in disordered graphene, Phys. Rev. B 92 (2015) 161411(R).

[108] S. Abdelouahed, A. Ernst, J. Henk, I.V. Maznichenko, I. Mertig, SpinSplit electronic states in graphene : Effects due to lattice deformation, Rashba effect, and adatoms by first principles, Phys. Rev. B 82 (2010) 125424 .

[109] D. Van Tuan, J.M. Marmolejo-Tejada, X. Waintal, B.K. Nikolić, S.O. Valenzuela, S. Roche, Spin Hall Effect and Origins of Nonlocal Resistance in Adatom-Decorated Graphene, Phys. Rev. Lett. 117 (2016) 176602 .

[110] Y. Cui, J. Gao, J. Zhao, D. Tan, Q. Fu, X. Bao, An Exchange Intercalation Mechanism for the Formation of a Two-Dimensional Si Structure Underneath Graphene, Nano Res. 5 (2012) 352-360.

[111] A. Sokolova, F. Kilchert, S. Link, A. Stöhr, U. Starke, M.A. Schneider, Sub-Monolayer Growth of Titanium, Cobalt and Palladium on Epitaxial Graphene, Ann. Phys. 529 (2017) 1700031.

[112] N.A. Anderson, M. Hupalo, D. Keavney, M.C. Tringides, D. Vaknin, Intercalated europium metal in epitaxial graphene on $\mathrm{SiC}$, Phys. Rev. Mat. 1 (2017) 054005.

[113] K.S. Kim, A.L. Walter, L. Moreschini, T. Seyller, K. Horn, E. Rotenberg, A. Bostwick, Coexisting massive and massless Dirac fermions in symmetry-broken bilayer graphene, Nat. Mater. 12 (2013) 887-892.

[114] J.H. Ho, C.L. Lu, C.C. Hwang, C.P. Chang, M.F. Lin, Coulomb excitations in AA- and AB-stacked bilayer graphites, Phys. Rev. B 74 (2006) 085406.

[115] B.R.K. Nanda, S. Satpathy, Strain and electric field modulation of the electronic structure of bilayer graphene, Phys. Rev. B 80 (2009) 165430.

[116] S. Ulstrup, M. Andersen, M. Bianchi, L. Barreto, B. Hammer, L. Hornekær, P. Hofmann, High Crystallinity and Decoupling of Graphene on a Metal : Reduced Coulomb Screening and Tunable pn-Junctions, arXiv :1404.6132 [cond-mat.mtrl-sci]. 
[117] S. Watcharinyanon, L.I. Johansson, A.A. Zakharov, C. Virojanadara, Studies of Li intercalation of hydrogenated graphene on $\mathrm{SiC}(0001)$, Surf. Sci. 606 (2012) 401-406.

[118] C. Jeon, H.-C. Shin, I. Song, M. Kim, J.-H. Park, J. Nam, D.-H. Oh, S. Woo, C.-C. Hwang, C.-Y. Park, J.R. Ahn, Opening and reversible control of a wide energy gap in uniform monolayer graphene, Scien. Rep. 3 (2013) 2725.

[119] L. Ye, M. Kang, J. Liu, F. von Cube, C.R. Wicker, T. Suzuki, C. Jozwiak, A. Bostwick, E. Rotenberg, D.C. Bell, L. Fu, R. Comin, J.G. Checkelsky, Massive Dirac fermions in a ferromagnetic kagome metal, Nature 555 (2018) 638-642.

[120] T. Valla, Z. Pan, in Physics and Applications of Graphene - Experiments, (Ed : S. Mikhailov), InTech, 2011, pp. 455-470.

[121] A.V. Fedorov, N.I. Verbitskiy, D. Haberer, C. Struzzi, L. Petaccia, D. Usachov, O.Y. Vilkov, D.V. Vyalikh, J. Fink, M. Knupfer, B. Büchner, A. Grüneis, Observation of a universal donor-dependent vibrational mode in graphene, Nat. Comm. 5 (2014) 3257.

[122] Z.-H. Pan, J. Camacho, M.H. Upton, A.V. Fedorov, C.A. Howard, M. Ellerby, T. Valla, Electronic Structure of Superconducting $\mathrm{KC}_{8}$ and Nonsuperconducting $\mathrm{Li}_{6}$ Graphite Intercalation Compounds : Evidence for a Graphene-Sheet-Driven Superconducting State, Phys. Rev. Lett. 106 (2011) 187002.

[123] C.C. Tsuei, J.R. Kirtley, Pairing symmetry in cuprate superconductors, Rev. Mod. Phys. 72 (2000) 969-1016.

[124] K. McElroy, R.W. Simmonds, J.E. Hoffman, D.-H. Lee, J. Orenstein, H. Eisaki, S. Uchida, J.C. Davis, Relating atomic-scale electronic phenomena to wave-like quasiparticle states in superconducting $\mathrm{Bi}_{2} \mathrm{Sr}_{2} \mathrm{CaCu}_{2} \mathrm{O}_{8+\delta}$, Nature 422 (2003) 592-596.

[125] R.S. Markiewicz, Van Hove Singularities and High- $T_{c}$ superconductivity : A Review, Int. J. Mod. Phys. B 05 (1991) 2037-2071. 
[126] R. Nandkishore, L.S. Levitov, A.V. Chubukov, Chiral superconductivity from repulsive interactions in doped graphene, Nat. Phys. 8 (2012) 158-163.

[127] G. Li, A. Luican, J.M.B. Lopes dos Santos, A.H. Castro Neto, A. Reina, J. Kong, E.Y. Andrei, Observation of Van Hove singularities in twisted graphene layers, Nat. Phys. 6 (2010) 109-113.

[128] S. Fang, E. Kaxiras, Electronic structure theory of weakly interacting bilayers, Phys. Rev. B 93 (2016) 235153.

[129] Y. Cao, V. Fatemi, A. Demir, S. Fhang, S.L. Tomarken, J.Y. Luo, J.D. Sanchez-Yamagishi, K. Watanabe, T. Taniguchi, E. Kaxiras, R.C. Ashoori, P. Jarillo-Herrero, Correlated insulator behaviour at halffilling in magic-angle graphene superlattices, Nature 556 (2018) 80-84.

[130] Y. Cao, V. Fatemi, S. Fhang, K. Watanabe, T. Taniguchi, E. Kaxiras, P. Jarillo-Herrero, Unconventional superconductivity in magic-angle graphene superlattices, Nature 556 (2018) 43-50.

[131] H. Zhang, Q. Fu, Y. Cui, D. Tan, X. Bao, Growth Mechanism of Graphene on $\mathrm{Ru}(0001)$ and $O_{2}$ Adsorption on the Graphene/Ru(0001) Surface, J. Phys. Chem. C 113 (2009) 8296-8301.

[132] E. Starodub, N.C. Bartelt, K.F. McCarty, Oxidation of Graphene on Metals, J. Phys. Chem. C 114 (2010) 5134-5140.

[133] P. Sutter, J.T. Sadowski, E.A. Sutter, Chemistry under Cover : Tuning Metal-Graphene Interaction by Reactive Intercalation, J. Am. Chem. Soc. 132 (2010) 8175-8179.

[134] L. Jin, Q. Fu, H. Zhang, R. Mu, Y. Zhang, D. Tan, X. Bao, Tailoring the Growth of Graphene on $\mathrm{Ru}(0001)$ via Engineering of the Substrate Surface, J. Phys. Chem. C 116 (2012) 2988-2993.

[135] A. Dong, Q. Fu, M. Wei, Y. Liu, Y. Ning, F. Yang, H. Bluhm, X. Bao, Facile oxygen intercalation between full layer graphene and $\mathrm{Ru}(0001)$ under ambient conditions, Surf. Sci. 634 (2015) 37-43.

[136] E. Gränäs, J. Knudsen, U.A. Schröder, T. Gerber, C. Busse, M.A. Arman, K. Schulte, J.N. Andersen, T. Michely, Oxygen Intercalation 
under Graphene on $\operatorname{Ir}(111)$ : Energetics, Kinetics, and the Role of Graphene Edges, ACS Nano 6 (2012) 9951-9963.

[137] R.S. Weatherup, L. D'Arsie, A. Cabrero-Vilatela, S. Caneva, R. Blume, J. Robertson, R. Schloegl, S. Hofmann, Long-Term Passivation of Strongly Interacting Metals with Single-Layer Graphene, J. Am. Chem. Soc. 137 (2015) 14358-14366.

[138] L. Bignardi, P. Lacovig, M.M. Dalmiglio, F. Orlando, A. Ghafari, L. Petaccia, A. Baraldi, R. Larciprete, S. Lizzit, Key role of rotated domains in oxygen intercalation at graphene on Ni(111), 2D Mat. 4 (2017) 025106 .

[139] L. Jin, Q. Fu, A. Dong, Y. Ning, Z. Wang, H. Bluhm, X. Bao, Surface Chemistry of $\mathrm{CO}$ on $\mathrm{Ru}(0001)$ under the Confinement of Graphene Cover, J. Phys. Chem. C 118 (2014), 12391-12398.

[140] M. Wei, Q. Fu, Y. Yang, W. Wei, E. Crumlin, H. Bluhm, X. Bao, Modulation of Surface Chemistry of CO on Ni(111) by Surface Graphene and Carbidic Carbon, J. Phys. Chem. C 119 (2015) 13590-13597.

[141] Y. Yao, Q. Fu, Y.Y. Zhang, X. Weng, H. Li, M. Chen, L. Jin, A. Dong, R. Mu, P. Jiang, L. Liu, H. Bluhm, Z. Liu, S.B. Zhang, X. Bao, Graphene cover-promoted metal-catalyzed reactions, Proc. Natl. Acad. Sci. USA 111 (2014) 17023-17028.

[142] R. Mu, Q. Fu, L. Jin, L. Yu, G. Fang, D. Tan, X. Bao, Visualizing Chemical Reactions Confined under Graphene, Angew. Chem. Int. Ed. 51 (2012) 4856-485.

[143] E. Grảnäs, M. Andersen, M.A. Arman, T. Gerber, B. Hammer, J. Schnadt, J.N. Andersen, T. Michely, J. Knudsen, CO Intercalation of Graphene on $\operatorname{Ir}(111)$ in the Millibar Regime, J. Phys. Chem. C 117 (2013) 16438-16447.

[144] X. Feng, S. Maier, M. Salmeron, Water Splits Epitaxial Graphene and Intercalates, J. Am. Chem. Soc. 134 (2012) 5662-5668.

[145] M. Ohtomo, Y. Sekine, S. Wang, H. Hibino, H. Yamamoto, Etchant-free graphene transfer using facile intercalation of alkanethiol self-assembled 
molecules at graphene/metal interfaces, Nanoscale 8 (2016) 1150311510.

[146] E.V. Rut'kov, A.Ya. Tontegode, M.M. Usufov, Evidence for a $C_{60}$ Monolayer Intercalated between a Graphite Monolayer and Iridium, Phys. Rev. Lett. 74 (1995) 758-760.

[147] A. Varykhalov, W. Gudat, O. Rader, Imaging Buried Molecules : Fullerenes Under Graphene, Adv. Mater. 22 (2010) 3307-3310.

[148] E. Monazami, L. Bignardi, P. Rudolf, P. Reinke, Strain Lattice Imprinting in Graphene by $C_{60}$ Intercalation at the Graphene/Cu Interface, Nano Lett. 15 (2015) 7421-7430.

[149] C. Riedl, C. Coletti, T. Iwasaki, A.A. Zakharov, U. Starke, Quasi-FreeStanding Epitaxial Graphene on SiC Obtained by Hydrogen Intercalation, Phys. Rev. Lett. 103 (2009) 246804.

[150] S. Oida, F.R. McFeely, J.B. Hannon, R.M. Tromp, M. Copel, Z. Chen, Y. Sun, D.B. Farmer, J. Yurkas, Decoupling graphene from $\mathrm{SiC}(0001)$ via oxidation, Phys. Rev. B 82 (2010) 041411.

[151] Y.Zhang, Q. Fu, Y. Cui, R. Mu, L. Jin, X. Bao, Enhanced reactivity of graphene wrinkles and their function as nanosized gas inlets for reactions under graphene, Phys. Chem. Chem. Phys. 15 (2013) 19042.

[152] Z.Y. Al Balushi, K. Wang, R.K. Ghosh, R.A. Vilá, S.M. Eichfeld, J.D. Caldwell, X. Qin, Y.-C. Lin, P.A. DeSario, G. Stone, S. Subramanian, D.F. Paul, R.M. Wallace, S. Datta, J.M. Redwing, J.A. Robinson, Twodimensional gallium nitride realized via graphene encapsulation, Nat. Mat. 15 (2016) 1166-1171.

[153] W. Choi, N. Choudhary, G.H. Han, J. Park, D. Akinwande, Y.H. Lee, Recent development of two-dimensional transition metal dichalcogenides and their applications, Mat. Today 20 (2017) 116-130.

[154] R.H. Friend, A.D. Yoffe, Electronic properties of intercalation complexes of the transition metal dichalcogenides, Adv. Phys. 36 (1987) $1-94$. 
[155] S. Balendhran, S. Walia, H. Nili, J.Z. Ou, S. Zhuiykov, R.B. Kaner, S. Sriram, M. Bhaskaran, K. Kalantar-zadeh, Two-Dimensional Molybdenum Trioxide and Dichalcogenides, Adv. Funct. Mater. 23 (2013) 3952-3970.

[156] K.F. Mak, C. Lee, J. Hone, J. Shan, T.F. Heinz, Atomically Thin $\mathrm{MoS}_{2}$ : A New Direct-Gap Semiconductor, Phys. Rev. Lett. 105 (2010) 136805 .

[157] A. Splendiani, L. Sun, Y. Zhang, T. Li, J. Kim, C.-Y. Chim, G. Galli, F. Wang, Emerging Photoluminescence in Monolayer $\mathrm{MoS}_{2}$, Nano Lett. 10 (2010) 1271-1275.

[158] C. Lee, Q. Li, W. Kalb, X.-Z. Liu, H. Berger, R.W. Carpick, J. Hone, Frictional Characteristics of Atomically Thin Sheets, Science $\mathbf{3 2 8}$ (2010) 76-80.

[159] B. Radisavljevic, A. Radenovic, J. Brivio, V. Giacometti, A. Kis, Single-layer $\mathrm{Mo}_{2}$ transistors, Nat. Nanotech. 6 (2011) 147-150.

[160] A. Kumar, P.K. Ahluwalia, Electronic structure of transition metal dichalcogenides monolayers $1 \mathrm{H}-\mathrm{M}_{2}(\mathrm{M}=\mathrm{Mo}, \mathrm{W} ; \mathrm{X}=\mathrm{S}, \mathrm{Se}, \mathrm{Te})$ from ab-initio theory : new direct band gap semiconductors, Eur. Phys. J. B 85 (2012) 18.

[161] S.K. Mahatha, K.D. Patel, K.S.R. Menon, Electronic structure investigation of $\mathrm{MoS}_{2}$ and $\mathrm{MoSe}_{2}$ using angle-resolved photoemission spectroscopy and ab initio band structure studies, J. Phys. Condens. Matter 24 (2012) 475504.

[162] Z.Y. Zhu, Y.C. Cheng,U. Schwingenschlögl, Giant spin-orbit-induced spin splitting in two-dimensional transition-metal dichalcogenide semiconductors, Phys. Rev. B 84 (2011) 153402.

[163] D. Xiao, G.-B. Liu, W. Feng, X. Xu, W. Yao, Coupled Spin and Valley Physics in Monolayers of $\mathrm{MoS}_{2}$ and Other Group-VI Dichalcogenides, Phys. Rev. Lett. 108 (2012) 196802.

[164] T. Eknapakul, P.D.C. King, M. Asakawa, P. Buaphet, R.-H. He, S.-K. Mo, H. Takagi, K.M. Shen, F. Baumberger, T. Sasagawa, S. Jungthawan, W. Meevasana, Electronic Structure of a Quasi-Freestanding Mo $S_{2}$ Monolayer, Nano Lett. 14 (2014) 1312-1316. 
[165] R. Bissessur, M.G. Kanatzidis, J.L. Schindler, C.R. Kannewurf, Encapsulation of polymers into $\mathrm{MoS}_{2}$ and metal to insulator transition in metastable $\mathrm{Mo}_{2}$, J. Chem. Soc. Chem. Commun. 0 (1993) 1582-1585.

[166] R. Kappera , D. Voiry , S.E. Yalcin , B. Branch , G. Gupta, A.D. Mohite, M. Chhowalla, Phase-engineered low-resistance contacts for ultrathin $\mathrm{Mo}_{2}$ transistors, Nat. Mater. 13 (2014) 1128-1134.

[167] B. Pal, A. Singh, S. Govinda, P. Mahale, A. Kumar, S. Thirupathaiah, H. Sezen, M. Amati, L Gregoratti, U.V. Waghmare, D.D. Sarma, Chemically exfoliated $\mathrm{Mo}_{2}$ layers : Spectroscopic evidence for the semiconducting nature of the dominant trigonal metastable phase, Phys. Rev. B 96 (2017) 195426.

[168] K.-K. Liu, W. Zhang, Y.-H. Lee, Y.-C. Lin, M.-T. Chang, C.-Y. Su, C.S. Chang, H. Li, Y. Shi, H. Zhang, C.-S. Lai, L.-J. Li, Growth of largearea and highly crystalline $\mathrm{MoS}_{2}$ thin layers on insulating substrates, Nano Lett. 12 (2012) 1538-1544.

[169] Y. Shi, W. Zhou, A.-Y. Lu, W. Fang, Y.-H. Lee, A.L. Hsu, S.M. Kim, K.K. Kim, H.Y. Yang, L.-J. Li, J.-C. Idrobo, J. Kong, van der Waals Epitaxy of $\mathrm{Mo}_{2}$ Layers Using Graphene As Growth Templates, Nano Lett. 12 (2012) 2784-2791.

[170] Y. Zhan, Z. Liu, S. Najmaei, P.M. Ajayan, J. Lou, Large-Area VaporPhase Growth and Characterization of $\mathrm{MoS}_{2}$ Atomic Layers on a $\mathrm{SiO}_{2} 2$ Substrate, Small 8 (2012) 966-971.

[171] K. Kang, S. Xie, L. Huang, Y. Han , P.Y. Huang, K.F. Mak, C.-J. Kim , D. Muller, J. Park, High-mobility three-atom-thick semiconducting films with wafer-scale homogeneity, Nature 520 (2015) 656-660.

[172] L.K. Tan, B. Liu, J.H. Teng, S. Guo, H.Y. Low, K.P. Loh, Atomic layer deposition of a MoS $S_{2}$ film, Nanoscale 6 (2014) 10584-10588.

[173] J.-G. Song, J. Park, W. Lee, T. Choi, H. Jung, C.W. Lee, S.-H. Hwang, J.M. Myoung, J.-H. Jung, S.-H. Kim, C. Lansalot-Matras, H. Kim , Layer-Controlled, Wafer-Scale, and Conformal Synthesis of Tungsten Disulfide Nanosheets Using Atomic Layer Deposition, ACS Nano 7 (2013) 11333-11340. 
[174] J.N. Coleman, M. Lotya, A. O'Neill, S.D. Bergin, P.J. King, U. Khan, K. Young, A. Gaucher, S. De, R.J. Smith, I.V. Shvets, S.K. Arora, G. Stanton, H.-Y. Kim, K. Lee, G.T. Kim, G.S. Duesberg, T. Hallam, J.J. Boland,J.J. Wang, J.F. Donegan, J.C. Grunlan, G. Moriarty, A. Shmeliov, R.J. Nicholls, J.M. Perkins, E.M. Grieveson, K. Theuwissen, D.W. McComb, P.D. Nellist, V. Nicolosi, Two-Dimensional Nanosheets Produced by Liquid Exfoliation of Layered Materials, Science 331 (2011) 568-571.

[175] Y. Jung, Y. Zhou, J.J. Cha, Intercalation in two-dimensional transition metal chalcogenides, Inorg. Chem. Front. 3 (2016) 452-463.

[176] M.A. Py, R.R. Haering, Structural destabilization induced by lithium intercalation in $\mathrm{Mo}_{2}$ and related-compounds, Can. J. Phys. 61 (1983) 76-84.

[177] G. Eda, T. Fujita, H. Yamaguchi, D. Voiry, M. Chen, M. Chhowalla, Coherent Atomic and Electronic Heterostructures of SingleLayer $\mathrm{MoS}_{2}$, ACS Nano 6 (2012) 7311-7317.

[178] M.A. Lukowski, A.S. Daniel, F. Meng, A. Forticaux, L. Li, S. Jin, Enhanced Hydrogen Evolution Catalysis from Chemically Exfoliated Metallic Mo $S_{2}$ Nanosheets, J. Am. Chem. Soc. 135 (2013) 10274-10277.

[179] J.S. Kim, J. Kim, J. Zhao, S. Kim, J.H. Lee, Y. Jin, H. Choi, B.H. Moon, J.J. Bae, Y.H. Lee, S.C. Lim, Electrical Transport Properties of Polymorphic Mo $S_{2}$, ACS Nano 10 (2016) 7500-7506.

[180] R.B. Somoano, V. Hadek, A. Rembaum, Alkali metal intercalates of molybdenum disulfide, J. Chem. Phys. 58 (1973) 697-701. 